\title{
Robust synthesis of two-dimensional metal dichalcogenides and their alloys by active chalcogen monomer supply
}

Kaihui Liu ( $\nabla$ khliu@pku.edu.cn )

State Key Laboratory for Mesoscopic Physics, Frontiers Science Center for Nano-optoelectronics, School of Physics, Peking University, Beijing 100871 https://orcid.org/0000-0002-8781-2495

Yonggang Zuo Institute of Physics, Chinese Academy of Sciences https://orcid.org/0000-0003-1262-6767

Can Liu

State Key Laboratory for Mesoscopic Physics, Frontiers Science Centre for Nanooptoelectronics, School of Physics, Peking University, Beijing, China https://orcid.org/0000-0001-5451-4144

\section{Liping Ding}

Institute for Basic Science

\section{Ruixi Qiao}

Peking University

Chang Liu

Peking University

\section{Ying Fu}

Institute of Physics, Chinese Academy of Sciences

Kehai Liu

Institute of Physics, Chinese Academy of Sciences

\section{Xu Zhou}

South China Normal University https://orcid.org/0000-0003-3318-8735

\section{Qinghe Wang}

Peking University

\section{Quanlin Guo}

Peking University

\section{Guodong Xue}

Peking University

\section{Jinhuan Wang}

Beijing Institute of Technology

\section{Hao Hong}

State Key Laboratory for Mesoscopic Physics, Collaborative Innovation Centre of Quantum Matter, School of Physics, Peking University 


\section{Muhong Wu}

Peking University https://orcid.org/0000-0003-3607-8945

\section{Dapeng Yu}

Institute for Quantum Science and Engineering and Department of Physics, South University of Science and Technology of China

\section{Enge Wang}

Peking University

\section{Xuedong Bai}

Institute of Physics, Chinese Academy of Sciences https://orcid.org/0000-0002-1403-491X

\section{Feng Ding}

Institute for Basic Science https://orcid.org/0000-0001-9153-9279

\section{Physical Sciences - Article}

Keywords: Two-dimensional transition metal dichalcogenides, alloys, chalcogen monomers supply

Posted Date: April 21st, 2021

DOI: https://doi.org/10.21203/rs.3.rs-411823/v1

License: (c) (i) This work is licensed under a Creative Commons Attribution 4.0 International License. Read Full License 

their alloys by active chalcogen monomer supply

3 Yonggang $\mathrm{Zuo}^{1,2 \#}$, Can Liu ${ }^{1 \#}$, Liping Ding ${ }^{3 \#}$, Ruixi Qiao ${ }^{4 \#}$, Chang $\mathrm{Liu}^{4}$, Ying Fu ${ }^{5}$, Kehai $\mathrm{Liu}^{5}, \mathrm{Xu}$

4 Zhou $^{6}$, Qinghe Wang ${ }^{1}$, Quanlin Guo $^{1}$, Guodong Xue ${ }^{1}$, Jinhuan Wang ${ }^{1}$, Hao Hong ${ }^{1}$, Muhong Wu ${ }^{4,7}$,

5 Dapeng $\mathrm{Yu}^{8}$, Enge Wang ${ }^{4,5,9}$, Xuedong Bai ${ }^{2,5 *}$, Feng Ding ${ }^{3 *}$, Kaihui Liu ${ }^{1,4 *}$

61 State Key Laboratory for Mesoscopic Physics, Frontiers Science Centre for Nano-

7 optoelectronics, School of Physics, Peking University, Beijing, China

$8 \quad{ }^{2}$ Beijing National Laboratory for Condensed Matter Physics, Institute of Physics, Chinese

9 Academy of Sciences, Beijing, China

$10{ }^{3}$ Centre for Multidimensional Carbon Materials, Institute for Basic Science, Ulsan, South Korea

$11{ }^{4}$ International Centre for Quantum Materials, Collaborative Innovation Centre of Quantum

12 Matter, Peking University, Beijing, China

135 Songshan Lake Materials Laboratory, Institute of Physics, Chinese Academy of Sciences, 14 Dongguan, China

$15{ }^{6}$ School of Physics and Telecommunication Engineering, South China Normal University, 16 Guangzhou, China

$17{ }^{7}$ Interdisciplinary Institute of Light-Element Quantum Materials and Research Center for Light-

18 Element Advanced Materials, Peking University, Beijing, China

$19{ }^{8}$ Shenzhen Institute for Quantum Science and Engineering, Southern University of Science and 20 Technology, Shenzhen, China

$21{ }^{9}$ School of Physics, Liaoning University, Shenyang, China

22 \# These authors contributed equally to this work

23 *Correspondence: khliu@pku.edu.cn, f.ding@unist.ac.kr, xdbai@iphy.ac.cn 
24 Two-dimensional (2D) transition metal dichalcogenides (TMDs), with their atomic 25 thicknesses, high carrier mobility, fast charge transfer, and intrinsic spin-valley couplings, 26 have been demonstrated one of the most appealing candidates for next-generation 27 electronic and optoelectronic devices ${ }^{1-3}$. The synthesis of TMDs with well-controlled 28 crystallinity, quality and composition is essential to fully realize their promising 29 applications ${ }^{4-13}$. Similar to that in III-V semiconductor synthesis, the precise precursor 30 supply is a precondition for controllable growth of TMDs. Although great efforts have been 31 devoted to modulate the transition metal supply, few effective methods of chalcogen feeding 32 control were developed ${ }^{14-21}$. Herein we report a strategy of using active chalcogen monomer supply to grow TMDs and their alloys in a robust and controllable manner. It is found that at a high temperature, the active chalcogen monomers (such as $\mathrm{S}$, Se, Te atoms or their mixtures) can be controllably released from metal chalcogenides and, thus, enable the synthesis of $\mathrm{TMDs}\left(\mathrm{MX}_{2}, \mathrm{M}=\mathrm{Mo}, \mathrm{W} ; \mathrm{X}=\mathrm{S}, \mathrm{Se}, \mathrm{Te}\right)$ with very high quality, e.g., $\mathrm{MoS}_{2}$ monolayers exhibit photoluminescent circular helicity of $\sim 92 \%$, comparable to the best exfoliated single-crystal flakes and close to the theoretical limit of unity. More intriguingly, a uniform quaternary TMD alloy with three different anions, i.e., $\operatorname{MoS}_{2(1-x-y)} \operatorname{Se}_{2 x} T_{2 y}$, was accomplished for the first time. Our mechanism study revealed that the active chalcogen monomers can bind and diffuse freely on a TMD surface, which enables the effective nucleation and reaction, quick chalcogen vacancy healing, and alloy formation during the growth. The chalcogen monomer supply strategy offers more degrees of freedom for the controllable synthesis of $2 \mathrm{D}$ compounds and their alloys, which will greatly benefit the development of high-end devices with desired 2D materials. 
It is well known that the controllable growth of multi-element bulk materials is generally much more challenging than that of single-element ones. For example, the synthesized first48 generation semiconductor, silicon, can have the extremely low impurity of $\sim 10^{-11}$ and is nearly 49 threading dislocation free, but the synthesized third-generation semiconductor, GaN, generally have a much higher impurity, $\sim 10^{-4}$, and a threading dislocation density of $\sim 10^{4}-10^{5} \mathrm{~cm}^{-2}\left(\mathrm{ref}^{22}\right)$.

51 Analogously for the growth of 2D materials, the as-grown graphene already has excellent properties, which is comparable to the samples exfoliated from natural crystals, and the measured carrier mobilities are close to the theoretical limit ${ }^{23}$, while the as-grown 2D compounds, such as TMDs, typically have lower quality than the natural crystals or the theoretical expectations ${ }^{24}$.

The main difficulty in controllable compounds' growth lies in the complicated feeding of several elements simultaneously during the growth process. Therefore, in the semiconductor industry, advanced and expensive techniques such as molecular beam epitaxy (MBE) and metal-organic chemical vapour deposition (MOCVD) have been developed to realize the precise control of multi-element supplies for the compound film growth.

The synthesis of high-quality TMD materials requires the precise feeding control of both the transition metal and chalcogen precursors as well. In the past decade, intensive efforts have been devoted to optimizing the feeding of metal precursors by thermal evaporation or molten-saltassisted evaporation of metal oxide ${ }^{14}$, decomposition of metal-organic precursor ${ }^{15}$, direct deposition of metal layers, and others ${ }^{16-18}$. Although some methods for controllable chalcogen feeding, such as using either elemental chalcogen or chalcogen compounds (i.e., heating sulfur powder, using $\mathrm{H}_{2} \mathrm{~S}$ gas and ammonium sulphide ${ }^{19-21}$ ), have also been developed, it turns out that the chalcogen feeding control is much less effective than metal feeding control, as indicated by the most challenging problem in TMD quality control that the most synthesized TMDs are rich with chalcogen vacancies ${ }^{25}$. Therefore, developing more effective chalcogen supply methods to enable the growth of high-quality TMDs is of critical importance. 
In this work, we propose to use a chalcogen monomer feeding method in controllable TMD growth because of the following advantages: $(\boldsymbol{i})$ the chalcogen monomers or atoms are generally very active than corresponding dimers or bulk and thus they can quickly react with metal precursors to form TMDs, (ii) the active chalcogen monomers can bind and quickly diffuse on a TMD surface to scavenge the vacancy defects effectively, which will greatly improve the quality of the TMDs and (iii) an active chalcogen monomer can react with a TMD and easily substitute a chalcogen atom and, thus, allow the synthesis of uniform TMD chalcogen alloys. However, as chalcogen of monomer state only exists at very high temperature $(>2500 \mathrm{~K})$ under normal circumstance ${ }^{26}$, the most used methods can't produce enough chalcogen monomers at the typical TMD growth temperature, which is generally less than $1273 \mathrm{~K}$. Herein, we developed an effective route to provide chalcogen monomer by heating a metal chalcogenide. The success of this approach lies in that the dangling bonds on the surface of metal chalcogenides are unstable and can be easily broken to release chalcogen atoms (monomers) under a relatively low temperature ${ }^{27}$, ${ }^{28}$, and the slowly released chalcogen atoms have a very low probability to react with each other to form dimers.

In our design, metal chalcogenide plate, $\mathrm{ZnS}$, which served as the source for $\mathrm{S}$ monomers, was directly placed above the growth substrates of sapphire or $\mathrm{SiO}_{2} / \mathrm{Si}$ (see Methods for details). The substrates were precoated with a thin layer of $\mathrm{Na}_{2} \mathrm{MoO}_{4}$ precursor to provide Mo source for $\mathrm{MoS}_{2}$ growth (Fig. 1a, left panel). A confined space with a narrow gap of $\sim 20 \mu \mathrm{m}$ was naturally formed between the $\mathrm{ZnS}$ and the growth substrates. At an elevated growth temperature (750$950{ }^{\circ} \mathrm{C}$ ), $\mathrm{S}$ monomers slowly released from the $\mathrm{ZnS}$ surface can quickly reach the substrate surface and the possibility of their combination into dimers or polymers in the gas phase is very low (Fig. 1a, middle panel). These $\mathrm{S}$ monomers will react with melted $\mathrm{Na}_{2} \mathrm{MoO}_{4}$ pellets on the substrate to form monolayer $\mathrm{MoS}_{2}$ (Fig. 1a, right panel). Indeed, the release of S monomers from the metal sulphide surface was observed long time $\mathrm{ago}^{29}$, and was unambiguously revealed by the in-situ mass spectroscopy in our experiment (Fig. 1b). It is important to note that the $\mathrm{S}$ 
monomers can't be obtained by sublimation of sulfur powders, where $\mathrm{S}_{2}$ dimers are the dominating species at thermal equilibrium ${ }^{30}$.

Since 2D materials are atomic thick and their growth only needs a trace amount of chalcogen atoms, the slowly released $\mathrm{S}$ monomers are sufficient for the successful synthesis of uniform monolayer $\mathrm{MoS}_{2}$ with typical domain sizes of $50-80 \mu \mathrm{m}$ and continuous film (Fig.1c and Extended Data Fig. 1). A series of characterizations unequivocally reveal the high quality of $\mathrm{MoS}_{2}$ fed by S monomer supply. High-angle annular dark field scanning transmission electron microscopy (HAADF-STEM) image exhibited the perfect hexagonal honeycomb lattice with both Mo and S atoms (Fig. 1d). The low-temperature photoluminescence (PL) spectra of the Smonomer-feeding-grown $\mathrm{MoS}_{2}$ (Fig. 1e, orange curve) has a characteristic neutral exciton $\left(\mathrm{X}^{0}\right)$ emission peak accompanied with a trion $\left(\mathrm{X}^{\mathrm{T}}\right)$ peak (believed to be caused by the $\mathrm{n}$-type doping from substrate ${ }^{31}$ ), but the $\mathrm{X}^{\mathrm{D}}$ peak (believed to be caused by $\mathrm{S}$ vacancy ${ }^{32}$ and was obvious in Spowder-feeding-grown samples, Fig. 1e, dark yellow curve) is nearly invisible, which clearly proves the high quality of the S-monomer-feeding-grown $\mathrm{MoS}_{2}$ samples.

In addition, its high quality can be further confirmed by the measured optical circular helicity, which was detected to be as high as $92 \%$ (Fig. 1f) and comparable with the best exfoliated flakes from high-quality natural crystals ${ }^{33}$. The circular helicity is directly related to the scattering between $\mathrm{K}$ and $\mathrm{K}$ ' valleys in the Brillouin zone of $\mathrm{MoS}_{2}$ whilst the defects will greatly enhance the inter-valley scattering and decrease circular helicity value. Thus, the near-unity circular helicity strongly proves the high quality of the as-grown $\mathrm{MoS}_{2}$ samples. Here, we would like to note that intrinsic quality information of $\mathrm{MoS}_{2}$ accessed by the optical helicity measurement is more reliable than that via electronic device measurements, where the device fabrication process, contact quality and device configuration will all bring great uncertainties in quality assessment ${ }^{34}$.

Our strategy on $\mathrm{MoS}_{2}$ growth by monomer feeding has also been proved to be applicable for the growth of other high-quality TMD materials. Six typical monolayer TMDs (Fig. 2a) have 
been successfully synthesized by simply replacing the transition metal sources (e.g., $\mathrm{Na}_{2} \mathrm{MoO}_{4}$ and $\mathrm{Na}_{2} \mathrm{WO}_{4}$ ) and chalcogenide plates (e.g., $\mathrm{ZnS}, \mathrm{ZnSe}$, and $\mathrm{ZnTe}$ ) (see Methods). The Raman and PL spectra of these obtained TMD samples demonstrated the successful synthesis of the $2 \mathrm{H}$ phase $\mathrm{MoS}_{2}, \mathrm{WS}_{2}, \mathrm{MoSe}_{2}, \mathrm{WSe}, \mathrm{MoTe}_{2}$ and the 1T' phase $\mathrm{WTe}_{2}$ and $\mathrm{MoTe}_{2}$ (Fig. 2c-e and Extended Data Fig. 2). It's worth noting that the formation of transition metal tellurides, e.g., $\mathrm{MoTe}_{2}$ and $\mathrm{WTe}$, are usually less favourable when chalcogen bulks applied ${ }^{35}$, due to their higher formation energy compared to the corresponding sulphide and selenide (Fig. 2b). Thanks to the introduction of active Te monomers, the synthesis of $\mathrm{WTe}_{2}$ and $\mathrm{MoTe}_{2}$ turns to be much easier because of the greatly reduced formation energy.

The chalcogen monomer feeding method has a unique advantage in the growth of TMD chalcogen alloys. Since the evaporation temperatures, saturated vapour pressures and reaction energies of S, Se and Te are significantly different, it is nearly impossible to form high-quality TMD alloys with more than two anion elements by traditional approaches ${ }^{36}$. Till now, there is no report on the successful growth of $\mathrm{MoS}_{2(1-x-y)} \operatorname{Se}_{2 x} \mathrm{Te}_{2 y}$ alloy. In our experiment, we applied a compressed plate mixed with different metal chalcogenide powders, i.e., ZnS, ZnSe, and ZnTe, to supply three kinds of chalcogen monomers (S, Se, and Te) simultaneously (Fig. 3a). The as-

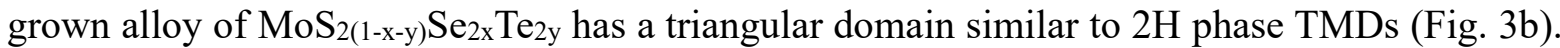
The X-ray photoelectron spectroscopy (XPS) unambiguously revealed the coexistence of S, Se, and Te atoms in the synthesized TMD alloy (Extended Data Fig. 3), and the energy dispersive Xray spectroscopy (EDS) as well as the STEM measurements further demonstrated the homogenous element distribution throughout the TMD alloy in both macro- and micro-scales, with no observable phase separation (Fig. 3e and Extended Data Fig. 4). Enlarged STEM image (Fig. 3f) further demonstrated the high crystallinity of the TMD alloy, and the distinct intensity distribution revealed the Mo, S, Se, and Te site occupancies according to the Z-contrast nature of HAADF image (Fig. 3g). Quantitative analysis of the doped Te and Se distribution was evaluated in a $32 \times 32 \mathrm{~nm}$ STEM image, and the statistical results match well with the binomial distribution 
model (Fig. 3h,i, Extended Data Fig. 5 and Methods), suggesting the random distribution of S,

149 Se and Te atoms in the TMD alloy.

Furthermore, one can easily tune the composition of the TMD alloys by controlling the growth temperature to vary the fluxes of S, Se and Te from the $\mathrm{ZnS}, \mathrm{ZnSe}$ and $\mathrm{ZnTe}$ composite. As the growth temperature increased, the PL peak shifted to longer wavelength (Fig. 3c), revealing the concentration of Se and Te atoms became higher in the alloy $\left(\mathrm{MoSe}_{2}\right.$ and $\mathrm{MoTe}_{2}$ have smaller bandgaps than $\mathrm{MoS}_{2}$ ). Meanwhile, in the Raman spectra, the $\mathrm{MoS}_{2}$-featured peaks gradually attenuated and the $\mathrm{MoTe}_{2}$-featured peaks gradually enhanced, while the $\mathrm{MoSe}_{2}-$ featured peaks enhanced first and then attenuated (Fig. 3d), which indicates that the heavy chalcogen atoms are being doped more into the $\mathrm{MoS}_{2}$ at higher temperature.

Finally, we try to understand the unique role of chalcogen monomer supply in the synthesis of high-quality TMDs and their complex alloys theoretically. We firstly explore the reactions of $\mathrm{Na}_{2} \mathrm{MoO}_{4}$ with sulfur monomers and dimers, respectively, by first-principles molecular dynamic (MD) simulations (Extended Data Fig. 6). The simulation results clearly demonstrated that sulfur monomers are more reactive to substitute the oxygen atoms in a $\mathrm{MoO}_{4}$ group of $\mathrm{Na}_{2} \mathrm{MoO}_{4}$ while most dimers are desorbed from the $\mathrm{Na}_{2} \mathrm{MoO}_{4}$ surface due to their less activity. By adding more $\mathrm{S}$ and $\mathrm{MoS}_{3}$ clusters to the $\mathrm{Na}_{2} \mathrm{MoO}_{4}$, the nucleation of $\mathrm{Mo}_{x} \mathrm{~S}_{y}$ clusters can also be clearly seen (Extended Data Fig. 7).

In compare with the bond-saturated $\mathrm{S}_{2} / \mathrm{Se}_{2} / \mathrm{Te}_{2}$ dimers, $\mathrm{S} / \mathrm{Se} / \mathrm{Te}$ monomers possess much higher adsorption energies on a TMD surface (for dimers $\mathrm{E}_{b}<0.8 \mathrm{eV}$, for monomers $\mathrm{E}_{\mathrm{b}}>1.5 \mathrm{eV}$ ) (Fig. 4b and Extended Data Fig. 8a). Therefore, one can expect a large number of $\mathrm{S} / \mathrm{Se} / \mathrm{Te}$ monomers to diffuse on the TMD surface during the whole growth process and the growth of

170 TMD is in a chalcogen monomer rich environment. Once a chalcogen monomer diffuse to the 171 vicinal area of a vacancy, the vacancy can be quickly healed by a highly exothermic reaction (Fig. 172 4a, Fig. 4c and Extended Data Fig. 8b). Therefore, the chalcogen vacancy density is greatly 
173 reduced as has been shown in the experimental results. To address the capacity of forming TMD

174 chalcogen alloys, we calculated the reaction energy of substituting a chalcogen atom in a TMD

175 by using chalcogen monomer, dimer, and bulk as references (Extended Data Fig. 9). It is clearly

176 shown that doping of Te dimer or bulk into $\mathrm{MoS}_{2} / \mathrm{MoSe}_{2}, \mathrm{~S}$ bulk into $\mathrm{MoSe}_{2}$, Se bulk into $\mathrm{MoS}_{2}$

177 are all difficult because of the near-zero or positive reaction energies. While, if chalcogen

178 monomer is used as the source of dopant, all doping reactions become more exothermic with

179 negative reaction energies, which implies the greatly improved capacity of forming chalcogen

180 TMD alloys.

181 This study clearly demonstrated that the high reactivity of chalcogen monomers can 182 significantly facilitate the TMD nucleation, chalcogen defects healing in the growth to greatly 183 improve the samples' quality and allow the formation of various chalcogen TMDs and their alloys.

184 The monomer supply should provide a new degree of freedom in modulating compound materials 185 and high-entropy 2D alloys, thus widening their potential applications in electronic, 186 optoelectronic and valleytronic devices. 


\section{References}

1 Ye, Z. L., Cao, T., O'Brien, K., Zhu, H. Y., Yin, X. B. et al. Probing excitonic dark states in single-layer tungsten disulphide. Nature 2014, 513, 214-218.

2 Novoselov, K. S., Mishchenko, A., Carvalho, A.,Castro Neto, A. H. 2D materials and van der Waals heterostructures. Science 2016, 353, aac9439.

3 Jauregui, L. A., Joe, A. Y., Pistunova, K., Wild, D. S., High, A. A. et al. Electrical control of interlayer exciton dynamics in atomically thin heterostructures. Science 2019, 366, 870875.

4 Chhowalla, M., Shin, H. S., Eda, G., Li, L. J., Loh, K. P. et al. The chemistry of twodimensional layered transition metal dichalcogenide nanosheets. Nat Chem 2013, 5, 263275.

5 Najmaei, S., Liu, Z., Zhou, W., Zou, X., Shi, G. et al. Vapour phase growth and grain boundary structure of molybdenum disulphide atomic layers. Nat Mater 2013, 12, 754-759.

6 van der Zande, A. M., Huang, P. Y., Chenet, D. A., Berkelbach, T. C., You, Y. M. et al. Grains and grain boundaries in highly crystalline monolayer molybdenum disulphide. Nat Mater 2013, 12, 554-561.

7 Huang, C. M., Wu, S. F., Sanchez, A. M., Peters, J. J. P., Beanland, R. et al. Lateral heterojunctions within monolayer MoSe2-WSe2 semiconductors. Nat Mater 2014, 13, 10961101.

8 Li, M. Y., Shi, Y. M., Cheng, C. C., Lu, L. S., Lin, Y. C. et al. Epitaxial growth of a monolayer $\mathrm{WSe}_{2}-\mathrm{MoS}_{2}$ lateral p-n junction with an atomically sharp interface. Science $\mathbf{2 0 1 5}$, $349,524-528$.

9 Li, S. S., Lin, Y. C., Zhao, W., Wu, J., Wang, Z. et al. Vapour-liquid-solid growth of monolayer $\mathrm{MoS}_{2}$ nanoribbons. Nat Mater 2018, 17, 535-542.

10 Sahoo, P. K., Memaran, S., Xin, Y., Balicas, L.,Gutierrez, H. R. One-pot growth of twodimensional lateral heterostructures via sequential edge-epitaxy. Nature 2018, 553, 63-67.

11 Aljarb, A., Fu, J. H., Hsu, C. C., Chuu, C. P., Wan, Y. et al. Ledge-directed epitaxy of continuously self-aligned single-crystalline nanoribbons of transition metal dichalcogenides. Nat Mater 2020, 19, 1300-1306.

12 Chowdhury, T., Kim, J., Sadler, E. C., Li, C. Y., Lee, S. W. et al. Substrate-directed synthesis of $\mathrm{MoS}_{2}$ nanocrystals with tunable dimensionality and optical properties. Nat 
Nanotechnol 2020, 15, 29-34.

13 Zeng, M. Q., Liu, J. X., Zhou, L., Mendes, R. G., Dong, Y. Q. et al. Bandgap tuning of twodimensional materials by sphere diameter engineering. Nat Mater 2020, 19, 528-533.

14 Zhou, J., Lin, J., Huang, X., Zhou, Y., Chen, Y. et al. A library of atomically thin metal chalcogenides. Nature 2018, 556, 355-359.

15 Kang, K., Xie, S., Huang, L., Han, Y., Huang, P. Y. et al. High-mobility three-atom-thick semiconducting films with wafer-scale homogeneity. Nature 2015, 520, 656-660.

16 Liu, L., Wu, J., Wu, L., Ye, M., Liu, X. et al. Phase-selective synthesis of $1 \mathrm{~T}^{\prime} \mathrm{MoS}_{2}$ monolayers and heterophase bilayers. Nat Mater 2018, 17, 1108-1114.

17 Shivayogimath, A., Thomsen, J. D., Mackenzie, D. M. A., Geisler, M., Stan, R. M. et al. A universal approach for the synthesis of two-dimensional binary compounds. Nat Commun 2019, 10, 2957.

18 Li, N., Wang, Q. Q., Shen, C., Wei, Z., Yu, H. et al. Large-scale flexible and transparent electronics based on monolayer molybdenum disulfide field-effect transistors. Nat Electron 2020, 3, 711-717.

19 Lin, H. H., Zhu, Q., Shu, D. H., Lin, D. J., Xu, J. et al. Growth of environmentally stable transition metal selenide films. Nat Mater 2019, 18, 602-607.

20 Dumcenco, D., Ovchinnikov, D., Sanchez, O. L., Gillet, P., Alexander, D. T. L. et al. Largearea $\mathrm{MoS}_{2}$ grown using $\mathrm{H}_{2} \mathrm{~S}$ as the sulphur source. 2 D Materials 2015, 2, 044005.

21 Lee, J. S., Choi, S. H., Yun, S. J., Kim, Y. I., Boandoh, S. et al. Wafer-scale single-crystal hexagonal boron nitride film via self-collimated grain formation. Science 2018, 362, 817821.

22 Zajac, M., Kucharski, R., Grabianska, K., Gwardys-Bak, A., Puchalski, A. et al. Basic ammonothermal growth of gallium nitride-state of the art, challenges, perspectives. Prog Cryst Growth Ch 2018, 64, 63-74.

23 Wang, L., Meric, I., Huang, P., Gao, Q., Gao, Y. et al. One-dimensional electrical contact to a two-dimensional material. Science 2013, 342, 614-617.

24 Kaasbjerg, K., Thygesen, K. S.,Jacobsen, K. W. Phonon-limited mobility in n-type singlelayer $\mathrm{MoS}_{2}$ from first principles. Phys Rev B 2012, 85, 115317.

25 Hong, J., Hu, Z., Probert, M., Li, K., Lv, D. et al. Exploring atomic defects in molybdenum 
disulphide monolayers. Nat Commun 2015, 6, 6293.

26 Berkowitz, J.,Marquart, J. R. Equilibrium Composition of Sulfur Vapor. J Chem Phys 1963, $39,275-283$.

27 Xu, X. Z., Zhang, Z. H., Qiu, L., Zhuang, J. N., Zhang, L. et al. Ultrafast growth of singlecrystal graphene assisted by a continuous oxygen supply. Nat Nanotechnol 2016, 11, 930935.

28 Liu, C., Xu, X. Z., Qiu, L., Wu, M. H., Qiao, R. X. et al. Kinetic modulation of graphene growth by fluorine through spatially confined decomposition of metal fluorides. Nat Chem 2019, 11, 730-736.

29 H.K. Pulker, E. J. An investigation of the evaporation process of dielectric materials. Thin Solid Film 1969, 4, 219-228.

30 Steudel, R., Steudel, Y.,Wong, M. W. Speciation and thermodynamics of sulfur vapor. Top Curr Chem 2003, 230, 117-134.

31 Mak, K. F., He, K. L., Lee, C., Lee, G. H., Hone, J. et al. Tightly bound trions in monolayer MoS2. Nat Mater 2013, 12, 207-211.

32 Carozo, V., Wang, Y., Fujisawa, K., Carvalho, B. R., McCreary, A. et al. Optical identification of sulfur vacancies: Bound excitons at the edges of monolayer tungsten disulfide. Sci Adv 2017, 3, e1602813.

33 Cao, T., Wang, G., Han, W., Ye, H., Zhu, C. et al. Valley-selective circular dichroism of monolayer molybdenum disulphide. Nat Commun 2012, 3, 887.

34 Liu, Y., Duan, X. D., Shin, H. J., Park, S., Huang, Y. et al. Promises and prospects of twodimensional transistors. Nature 2021, 591, 43-53.

35 Sung, J. H., Heo, H., Si, S., Kim, Y. H., Noh, H. R. et al. Coplanar semiconductor-metal circuitry defined on few-layer MoTe2 via polymorphic heteroepitaxy. Nat Nanotechnol 2017, $12,1064-1071$.

36 Mann, J., Ma, Q., Odenthal, P. M., Isarraraz, M., Le, D. et al. 2-Dimensional transition metal dichalcogenides with tunable direct band gaps: $\mathrm{MoS}_{2(1-\mathrm{x})} \mathrm{Se}_{2 \mathrm{x}}$ monolayers. Adv Mater 2014, 26, 1399-1404. 
276

277

278

279

280

281

282

283

284

285

286

287

288

289

290

291

292

293

294

295

296

297

298

299

Acknowledgements: This work was supported by the National Natural Science Foundation of China (52025023, 51991342, 52021006, 51991344 and 11888101), The Key R\&D Program of Guangdong Province (2020B010189001, 2019B010931001 and 2018B030327001), the Pearl River Talent Recruitment Program of Guangdong Province (2019ZT08C321), the Strategic Priority Research Program of Chinese Academy of Sciences (XDB33000000 and XDB33030200), Beijing Natural Science Foundation (JQ19004), Beijing Excellent Talents Training Support (2017000026833ZK11), National Postdoctoral Program for Innovative Talents (BX20190016) and China Postdoctoral Science Foundation (2019M660280, 2019M660281 and 2020T130022). We acknowledge the Electron Microscopy Laboratory in Peking University for the use of electron microscopy.

\section{Methods:}

\section{Growth of TMDs and their alloys.}

The substrate of sapphire or $\mathrm{SiO}_{2} / \mathrm{Si}$ was first pretreated by $\mathrm{O}_{2}$ plasma, followed by the spincoating process of $\mathrm{Na}_{2} \mathrm{MoO}_{4}$ or $\mathrm{Na}_{2} \mathrm{WO}_{4}$ aqueous solution with optimized concentrations $\left(\mathrm{Na}_{2} \mathrm{MoO}_{4}\right.$ of 12, 18, and $6 \mathrm{mg} / \mathrm{mL}$ for $\mathrm{MoS}_{2}, \mathrm{MoSe}_{2}$, and $\mathrm{MoTe}_{2}$ growth, $\mathrm{Na}_{2} \mathrm{WO}_{4}$ of 20, 30 and $12 \mathrm{mg} / \mathrm{mL}$ for $\mathrm{WS}_{2}, \mathrm{WSe}_{2}$, and $\mathrm{WTe}_{2}$ growth, respectively). After dried in Ar atmosphere, the chalcogenide crystal plate ( $\mathrm{ZnS}, \mathrm{ZnSe}$, or $\mathrm{ZnTe}$ ) was placed directly above the substrate and then loaded into the CVD furnace together. The chamber of furnace was flushed with $\operatorname{Ar}(100 \mathrm{sccm})$ and heated to the optimized growth temperature $\left(\sim 780{ }^{\circ} \mathrm{C}, \sim 930{ }^{\circ} \mathrm{C}, \sim 800{ }^{\circ} \mathrm{C}, \sim 820^{\circ} \mathrm{C}, \sim 750\right.$ $800{ }^{\circ} \mathrm{C}, \sim 800{ }^{\circ} \mathrm{C}$ and $\sim 780{ }^{\circ} \mathrm{C}$ for $\mathrm{MoS}_{2}, \mathrm{WS}_{2}, \mathrm{MoSe}_{2}, \mathrm{WSe}, 2 \mathrm{H}-\mathrm{MoTe}_{2}, 1 \mathrm{~T}{ }^{\prime}-\mathrm{MoTe}_{2}$ and $1 \mathrm{~T}{ }^{\prime}-$ $\mathrm{WTe}_{2}$, respectively). During the growth process, the system pressure was kept at $\sim 120 \mathrm{~Pa}$ and the growth duration was set as 10-60 minutes. After growth, the system was naturally cooled down to room temperature. Similar growth conditions were applied to the TMD alloy growth, wherein the major difference lies in the use of a chalcogenide mixture plate.

\section{Characterizations of TMDs and their alloys.}


Optical measurements. Optical images were taken with an Olympus BX51M microscope. Raman and PL spectra were measured by a customer-designed optical system with the excitation wavelength of $532 \mathrm{~nm}$ and the power of $\sim 1 \mathrm{~mW}$. Low-temperature PL spectra were obtained at $10 \mathrm{~K}$ by optical cryostat (Montana Instruments) with the laser excitation wavelength of $532 \mathrm{~nm}$. Circular-polarization-resolved PL measurements were performed under near-resonant excitation of $633 \mathrm{~nm}$ at $10 \mathrm{~K}$. The circularly polarized light was generated by using a super-achromatic quarter-wave plate (Thorlabs SAQWP05M-1700) and the photoluminescence was analyzed through the same quarter-wave plate and a linear-polarizer. We define the degree of PL circular helicity $(\eta)$, which reflects the valley polarization, as $\eta=\left(\operatorname{PL}\left(\sigma^{+}\right)-\operatorname{PL}\left(\sigma^{-}\right)\right) /\left(\operatorname{PL}\left(\sigma^{+}\right)+\operatorname{PL}\left(\sigma^{-}\right)\right)$.

$X P S, M S, E D S, S T E M$ characterizations. XPS measurements were performed using an ESCALAB 250X system (Thermo Fisher Scientific) and excited by monochromatic Al Ka radiation. Mass spectrometer (Hiden HR20) attached with temperature-programmed decomposition (TPD) was used to in-situ detect and analyze the released gas in inert atmosphere. EDS and STEM experiments were performed in FEI Titan Themis G2 300 operated at $300 \mathrm{kV}$ and in Nion U-HERMES200 at $60 \mathrm{kV}$ for element analysis and characterizing atomic structures of samples.

\section{Statistical Se and Te atoms distribution in the quaternary $\mathrm{MoS}_{2(1-x-y)} \operatorname{Se}_{2 x} \mathrm{Te}_{2 \mathrm{y}}$ alloy.}

To evaluate the distribution of Se and Te atoms in our quaternary alloy, statistical analysis was conducted in a $32 \times 32 \mathrm{~nm}$ STEM image (Extended Data Fig. 5a). The atoms in the image were sorted into metal atom $(\mathrm{M})$ sites and chalcogen atom $\left(\mathrm{X}_{2}\right)$ sites. The intensity histogram of all $\mathrm{X}_{2}$ sites shows three peaks, which are assigned to be "S site", "Se site" and "Te site" regions, respectively, according to the Z-contrast nature of STEM image (Extended Data Fig. 5b). Note that every $\mathrm{X}_{2}$ site in STEM image is actually a projection of two overlapped $\mathrm{X}$ atoms along the electron beam direction, "S site" denotes both $\mathrm{X}$ atoms are $\mathrm{S}$, "Se site" means two possible configurations: Se-S or Se-Se, while "Te site" stands for Te-S, Te-Se, or Te-Te. According to the overall statistical result, $P(S)$, the probability for an $\mathrm{X}_{2}$ site to be "S site", is 
$9122 /(9122+2417+771)=0.741$. Similarly, the probability of "Se site" and "Te site" denoting as $P(\mathrm{Se})$ and $P(\mathrm{Te})$, are 0.196 and 0.063 , respectively.

Assume that Se and Te are randomly distributed in the $\mathrm{X}_{2}$ sites, the overall probability of $P(\mathrm{Se})$ and $P(\mathrm{Te})$ will also be valid to every $\mathrm{X}_{2}$ site. Thus, the probability of Se distribution could be calculated by the binomial distribution

$$
f(k ; N, P(S e))=\frac{N !}{k !(N-k) !} P(S e)^{k}(1-P(S e))^{N-k}
$$

where $N$ is the total $\mathrm{X}_{2}$ site number of a region and $k$ is the "Se site" number found in it. The same analysis can be applied to the Te case. To verify this random-distribution assumption, the entire image was cut into identical $30 \times 30$ parts, each of which comprised about $14 \mathrm{X}_{2}$ sites. The statistical histogram of "Te site" and "Se site" for these 900 parts are shown in Fig. 3i and Extended Data Fig. 5d), which match well with the purple dotted lines calculated by the binomial distribution, suggesting the random distribution of Te and Se atoms in our quaternary alloy.

In addition, there is a relationship between the probability $P$ and the atom concentration $c$ :

$$
\begin{gathered}
P(S)=c(S)^{2} \\
P(S e)=2 c(S e) c(S)+c(S e)^{2} \\
c(S)+c(S e)+c(T e)=1
\end{gathered}
$$

from the probability data and these equations, the concentrations of S, Se and Te were extracted to be $86.1 \%, 10.7 \%, 3.2 \%$, respectively, which is very close to the concentrations extracted from the preceding EDS spectrum $(84.0 \%, 12.7 \%$, and 3.3\%, respectively, as mentioned in Extended Data Fig. 4) and confirms our statistical conclusion.

\section{Computational details.}

All the density functional theory (DFT) calculations were implemented by the Vienna Ab Initio Simulation Package (VASP) $)^{37,38}$, with projector-augmented wave (PAW) ${ }^{39}$ method describing the interaction between valence electrons and ion cores. The Perdew-Burke-Ernzerhof (PBE $)^{40,}$ ${ }^{41}$ exchange-correlation functional was used to describe the interaction between electrons. A plane 
wave basis set with a cutoff energy of $450 \mathrm{eV}$ was adopted. All the structures were fully relaxed, and the convergence criteria for energy and force were set at $10^{-5} \mathrm{eV}$ and $10^{-2} \mathrm{eV} / \AA$, respectively. The Brillion zone is sampled by $1 \times 1 \times 1$ grid meshes. A vacuum spacing larger than $15 \AA$ was set to avoid the interaction between neighboring images along the non-periodic direction. The energy barriers were calculated by using the climbing image nudged elastic band (CI-NEB) method ${ }^{42}$ with a force threshold of $-0.02 \mathrm{eV} / \AA$.

\section{Theoretical calculations on initiated nucleation of $\mathrm{MoS}_{2}$ by sulfur monomer or dimer supply.}

Based on the MD simulations, the sulfidation processes of $\mathrm{Na}_{2} \mathrm{MoO}_{4}$ are analyzed at atomic-level. Here we consider the sulfur source in the form of $\mathrm{S}$ monomers and $\mathrm{S}_{2}$ dimers respectively. As shown in Extended Data Fig. 6, eight $\mathrm{S}$ monomers and four $\mathrm{S}_{2}$ dimers were deposited on the $\mathrm{NaMoO}_{4}$ surface during the MD trajectories. The MD simulations clearly showed that $\mathrm{S}$ monomers are more active and can easily substitute the oxygen atoms of $\mathrm{Na}_{2} \mathrm{MoO}_{4}$, resulting in $\mathrm{SO}$ or $\mathrm{SO}_{2}$ molecules in gas phase and Mo-S bond formation in the liquid $\mathrm{Na}_{2} \mathrm{MoS}_{x} \mathrm{O}_{4-x}$ (green circle). This proves that $\mathrm{S}$ monomers released from the $\mathrm{ZnS}$ surface are more reactive in the growth of TMDs. Adding more $\mathrm{S}$ monomers to the $\mathrm{Na}_{2} \mathrm{MoO}_{4}$ surface (Extended Data Fig. 7a-b), the aggregation of Mo and S atoms during the MD simulation is clearly seen and the clusters of $\mathrm{MoS}_{3} \mathrm{O}_{x}$ are formed on the surface (Extended Data Fig. 7c-d). To demonstrate the nucleation of $\mathrm{MoS}_{2}$, we added a few $\mathrm{MoS}_{3}$ molecules on the substrate and large $\mathrm{Mo}_{x} \mathrm{~S}_{y}$ clusters (green circle) were formed during the MD simulation (Extended Data Fig. 7e-f). In summary, we have demonstrated the mechanism of TMD growth by the density-functional-theory-based MD simulations. Firstly, the active $\mathrm{S}$ monomers replace $\mathrm{O}$ atoms in $\mathrm{Na}_{2} \mathrm{MoO}_{4}$. Then, the high concentration of S substitutes in the $\mathrm{Na}_{2} \mathrm{MoO}_{4}$ will lead to the formation of $\mathrm{Mo}_{x} \mathrm{~S}_{y}$ clusters on the surface of the substrate. Finally, the aggregation of $\mathrm{Mo}_{x} \mathrm{~S}_{y}$ clusters results in the nucleation of $\mathrm{MoS}_{2}$ on the $\mathrm{Na}_{2} \mathrm{MoO}_{4}$ surface.

\section{Calculations of formation energies of different transition metal dichalcogenides.}


377 To understand the relative stability of these TMDs $\left(\mathrm{MX}_{2}, \mathrm{M}=\mathrm{Mo}, \mathrm{W} ; \mathrm{X}=\mathrm{S}, \mathrm{Se}, \mathrm{Te}\right)$, the 378 formation energies of these TMDs were calculated by using

$$
E_{f}=E_{M X_{2}}-\mu_{M}-2 \mu_{X},
$$

where $E_{M X_{2}}$ is the energy of the $\mathrm{MX}_{2}, \mu_{M}$ and $\mu_{X}$ are the energies of $\mathrm{M}$ in the form of bulk and $\mathrm{X}$ atoms using monomer or bulk as references, respectively. These results on formation energies (Fig. 2b) clearly show that $\mathrm{MS}_{2}$ and $\mathrm{MSe}_{2}$ are highly stable, while $\mathrm{MTe}_{2}$ might hardly to be synthesized due to the high formation energies, which agree well with the most experimental results of TMD synthesis.

\section{Reaction energies of ternary TMD alloys obtained by $X(X=S$, Se, Te $)$ doped $M_{0} X_{2}$.}

In order to confirm the possibility of forming $\mathrm{MoS}_{2(1-x-y)} \operatorname{Se}_{2 x} \mathrm{Te}_{2 y}$ alloy, we calculated the reaction energy $\left(E_{R}\right)$ of $X(X=S, S e, T e)$ doped $\mathrm{MoX}_{2}(X=S, S e, T e)$. Here, we take ternary alloy $\mathrm{MoSe}_{\mathrm{x}} \mathrm{S}_{2-\mathrm{x}}$ as an example. Its reaction energy can be calculated by using

$$
E_{R}=E_{\text {MoSe }_{x} S_{2-x}}-E_{M o S_{2}}+x \mu_{S}-x \mu_{S e},
$$

Where $\mathrm{E}_{\mathrm{MoSe}_{\mathrm{xS}}-\mathrm{x}}$ and $\mathrm{E}_{\mathrm{MoS}_{2}}$ are the energies of ternary alloy $\mathrm{MoSe}_{\mathrm{x}} \mathrm{S}_{2-\mathrm{x}}$ and perfect $\mathrm{MoS}_{2}$, respectively. $\mu_{\mathrm{S}}$ and $\mu_{\mathrm{Se}}$ are the energies of S and Se atoms which are in the form of bulk, dimer and monomer, respectively. The obtained different formation energies are shown in Extended Data Fig. 9. It is found that the monomers of S, Se and Te monomers can be easily doped into $\mathrm{MoX}_{2}$ with reaction energies less than $-2.0 \mathrm{eV}$. While the $\mathrm{Te}_{2}$ could hardly be used to dope $\mathrm{MoS}_{2}$ or $\mathrm{MoSe}_{2}$ because of the near zero reaction energy. If bulk dopants are employed, only $\mathrm{S} / \mathrm{Se}$ can easily dope the MoTe2. This result further confirms the advantage of using $\mathrm{S} / \mathrm{Se} / \mathrm{Te}$ monomer to grow $\mathrm{MoS}_{2(1-x-y)} \mathrm{Se}_{2 \mathrm{x}} \mathrm{Te}_{2 \mathrm{y}}$ alloys. 


\section{References}

39937 Kresse, G.,Hafner, J. Ab-initio molecular-dynamics for open-shell transition-metals. Phys 400 Rev $B$ 1993, 48, 13115-13118.

38 Kresse, G.,Furthmuller, J. Efficiency of ab-initio total energy calculations for metals and semiconductors using a plane-wave basis set. Comp Mater Sci 1996, 6, 15-50.

40339 Kresse, G.,Hafner, J. 14251; G. Kresse, J. Furthmüller. Phys. Rev. B 1996, 54, 11169-11186.

40440 Perdew, J. P., Burke, K.,Ernzerhof, M. Generalized gradient approximation made simple. 405 Phys Rev Lett 1996, 77, 3865-3868.

40641 Blochl, P. E. Projector augmented-wave method. Phys Rev B 1994, 50, 17953-17979.

40742 Henkelman, G., Uberuaga, B. P.,Jonsson, H. A climbing image nudged elastic band method 408 for finding saddle points and minimum energy paths. J Chem Phys 2000, 113, 9901-9904. 

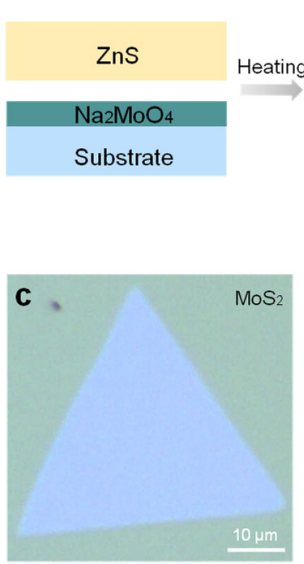

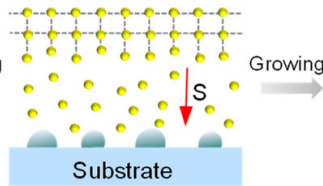

Substrate

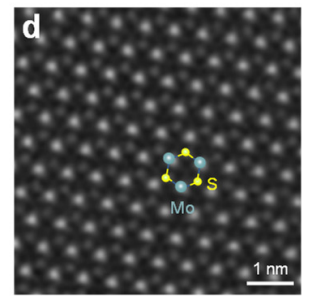

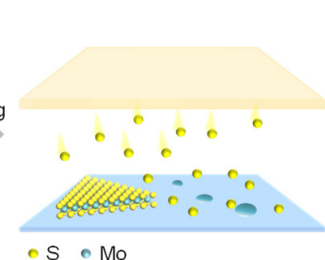

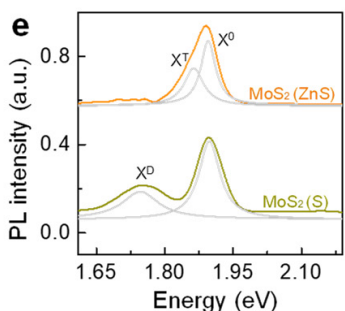

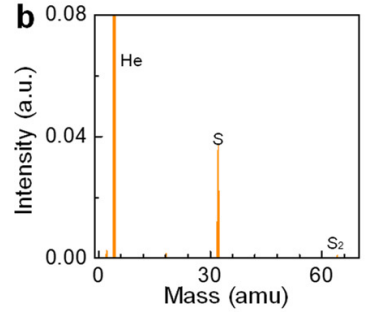

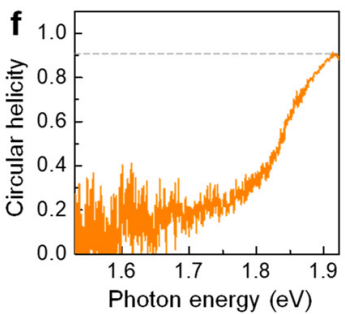

411 Figure 1 | Growth of high-quality monolayer MoS $\mathbf{2}$ by sulfur monomer supply. a, Schematic
412 of sulfur (S) monomer supply for the growth of $\mathrm{MoS}_{2}$. S monomers are released from the surface

413 of $\mathrm{ZnS}$ under high temperature, then confined in the narrow space and react with $\mathrm{Na}_{2} \mathrm{MoO}_{4}$ to

414 from $\mathrm{MoS}_{2}$. b, In-situ mass spectrum of $\mathrm{ZnS}$ annealed at $1000{ }^{\circ} \mathrm{C}$. The intense peak at the mass

415 of 32 clearly proves the dominating release of S monomers. The measurements were carried out

416 with carrier gas of $\mathrm{He}$ and the data was subtracted by background. c, Optical image of as-grown

417 monolayer $\mathrm{MoS}_{2}$ domain on sapphire. d, Atomic-resolved HAADF-STEM image of the prepared

$418 \mathrm{MoS}_{2}$, revealing the high crystallinity of $\mathrm{MoS}_{2}$ without detectable $\mathrm{S}$ vacancies. e, Low-

419 temperature (10 K) PL spectra of $\mathrm{MoS}_{2}$ samples fed by S monomer (orange curve) and S powder

420 (dark yellow curve), respectively. Three typical features, $\mathrm{X}^{0}, \mathrm{X}^{\mathrm{T}}$, and $\mathrm{X}^{\mathrm{D}}$ peaks assign to neutral

421 exciton, trion, and defect state emission peaks, respectively. The absence of $\mathrm{X}^{\mathrm{D}}$ peak confirmed

422 the high quality of $\mathrm{MoS}_{2}$ grown by S monomer supply. f, The circular dichroism PL spectrum

423 measured at $10 \mathrm{~K}$. The near-unity polarization of $\mathrm{MoS}_{2}$ on sapphire indicates the high optical

424 quality. 
a
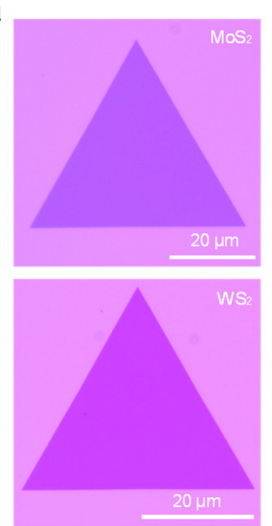
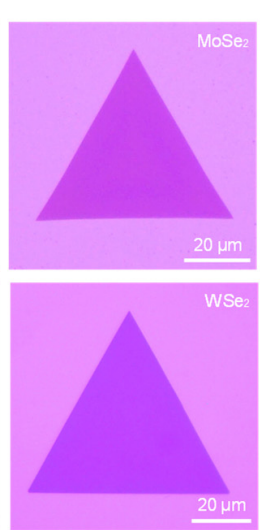
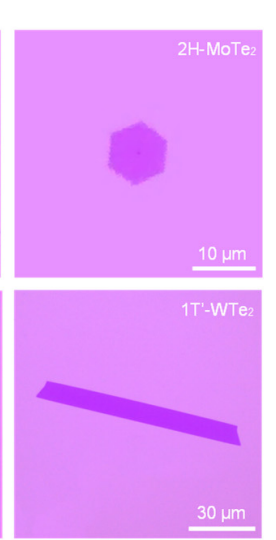

b
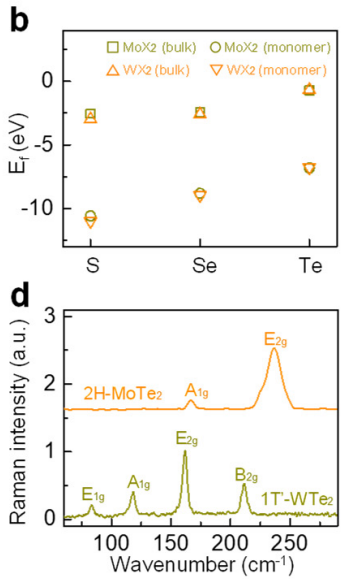
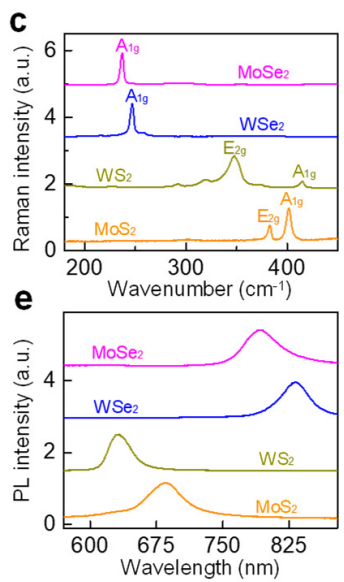

Figure 2 | Universal growth of diverse TMDs by chalcogen monomer supply. a, Optical

427 images of the representative TMDs, including $2 \mathrm{H}$ phase $\mathrm{MoS}_{2}, \mathrm{MoSe}_{2}, \mathrm{MoTe}_{2}, \mathrm{WS}_{2}, \mathrm{WSe}_{2}$ and

428 1T' phase $\mathrm{WTe}_{2}$. $\mathbf{b}$, The calculated formation energy $\left(\mathrm{E}_{\mathrm{f}}\right)$ of the six representative TMDs. When 429 chalcogen bulks are supplied as precursors, the formation of transition metal tellurides in relative

430 to their corresponding sulphide and selenide are less favourable due to their high formation 431 energy (-0.73 and $-0.68 \mathrm{eV} / \mathrm{unit}$ for $\mathrm{MoTe}_{2}$ and $\mathrm{WTe}_{2}$, respectively). While it becomes highly

432 favourable when Te monomers are applied. c-e, The corresponding Raman (c,d) and PL (e)

433 spectra of TMDs in (a). All spectra were vertically shifted for clarity. 

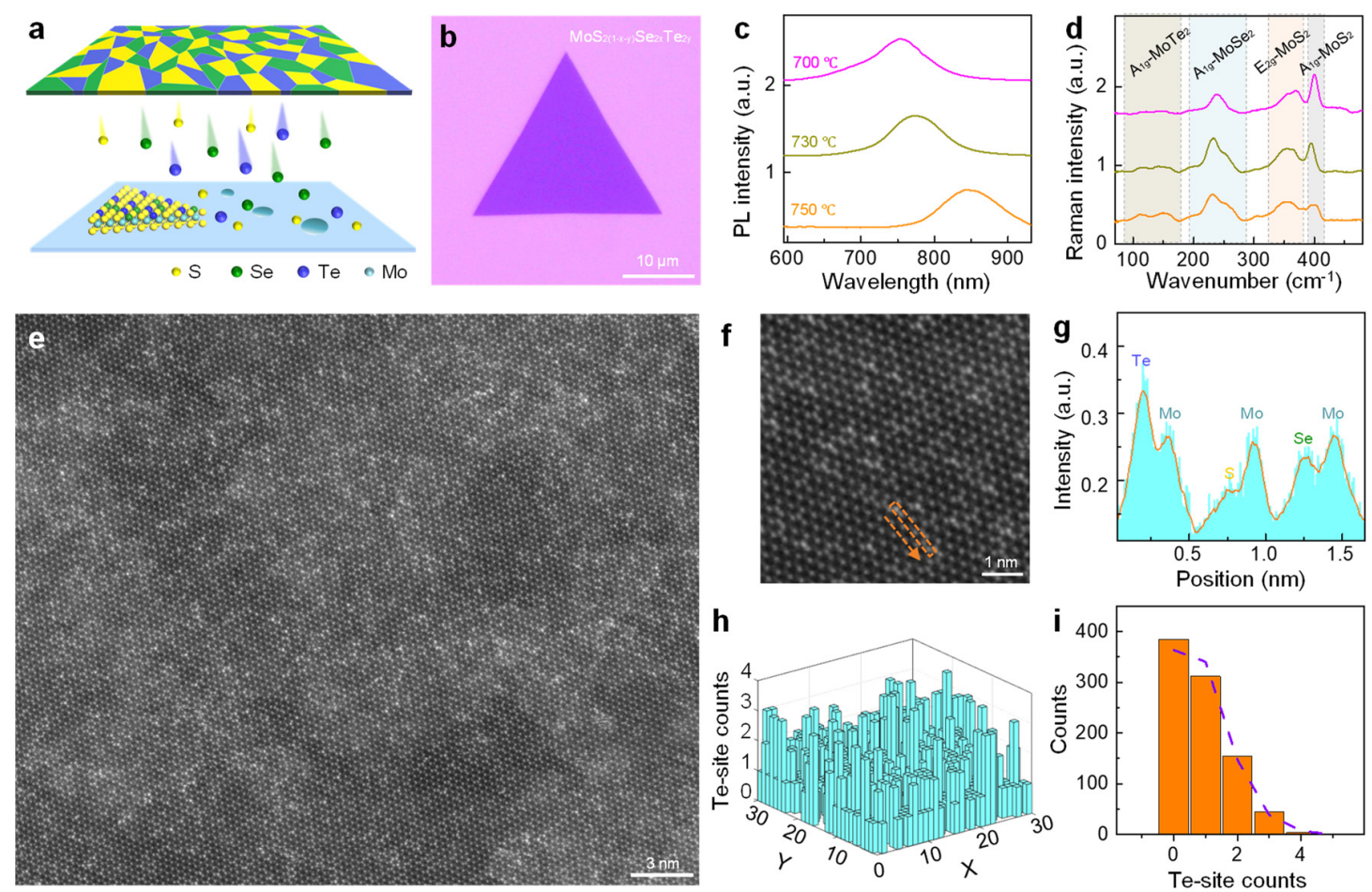

Figure 3 | Growth and characterization of quaternary TMD alloy. a, Schematic diagram of quaternary alloy growth using a compressed plate mixed with chalcogenide powders of $\mathrm{ZnS}$, $\mathrm{ZnSe}$, and ZnTe. b, Optical image of $\mathrm{MoS}_{2(1-\mathrm{x}-\mathrm{y})} \mathrm{Se}_{2 \mathrm{x}} \mathrm{Te}_{2 \mathrm{y}}$ domain on $\mathrm{SiO}_{2} / \mathrm{Si}$ substrate. c,d, PL (c) and Raman (d) spectra of the $\mathrm{MoS}_{2(1-\mathrm{x}-\mathrm{y})} \mathrm{Se}_{2 \mathrm{x}} \mathrm{Te}_{2 \mathrm{y}}$ sample grown at different temperatures. As the growth temperature increased, the PL peak position showed a clear red shift. The intensity of $\mathrm{MoS}_{2}$-like $\mathrm{E}_{2 \mathrm{~g}}\left(\sim 380 \mathrm{~cm}^{-1}\right)$ and $\mathrm{A}_{1 \mathrm{~g}}\left(\sim 400 \mathrm{~cm}^{-1}\right)$ was reduced while the $\mathrm{MoTe}_{2}$-like $\mathrm{A}_{1 \mathrm{~g}}\left(\sim 150 \mathrm{~cm}^{-}\right.$ $\left.{ }^{1}\right)$ increased and $\mathrm{MoSe}_{2}$-like $\mathrm{A}_{\lg }\left(\sim 240 \mathrm{~cm}^{-1}\right)$ increased first and then reduced. e,f, STEM images 442 of the $\mathrm{MoS}_{2(1-x-y)} \mathrm{Se}_{2 x} \mathrm{Te}_{2 y}$, demonstrating the high crystallinity of quaternary alloy. $\mathbf{g}$, Intensity 443 profiles along the labeled orange dotted box in (f), which highlights the occupancies of Mo, S, 444 Se, and Te sites. $\mathbf{h}$, The Te-site distribution in a $32 \times 32 \mathrm{~nm}$ STEM image of the quaternary alloy. 445 The image was divided into $30 \times 30$ parts. i, The corresponding statistical histogram of Te-site 446 counts in each parts of the image. It shows a well binomial distribution feature (purple dotted 447 line), revealing the random distribution of Te atoms. 

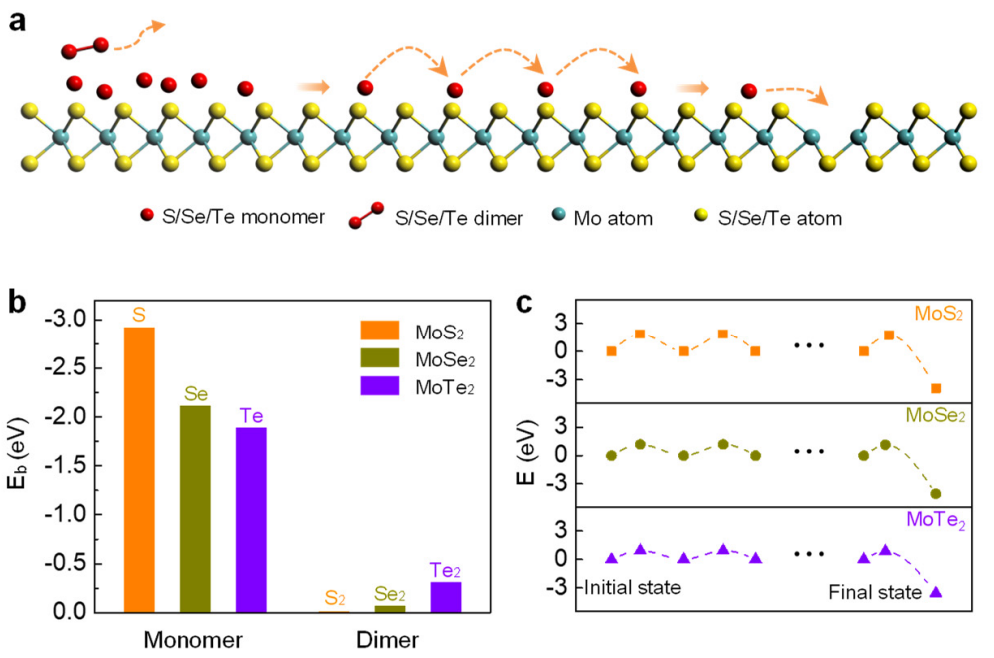

449 Figure 4 | Growth Mechanism with chalcogen monomer supply in $\mathbf{M o X}_{2}(X=S, S e, T e)$,

450 Schematic diagram of adsorption, diffusion, and vacancy healing of chalcogen monomer on $451 \mathrm{MoX}_{2}$ surface. $\mathbf{b}$, The binding energies of monomers and dimers on MoX 2 surface. The much 452 higher energy of monomers facilitates their better adsorption on the TMD surface than dimers. c, 453 The energy profiles of vacancy healing for $\mathrm{MoX}_{2}$ surface by using chalcogen monomers. The 454 relatively small energy barriers of chalcogen monomer diffusion and the highly exothermic 455 reaction at the vacancy both accelerate the self-healing of MoX 2. 


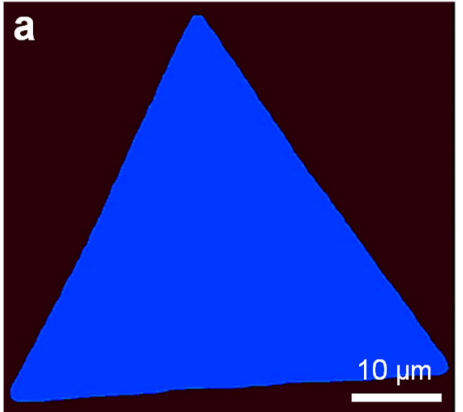

456
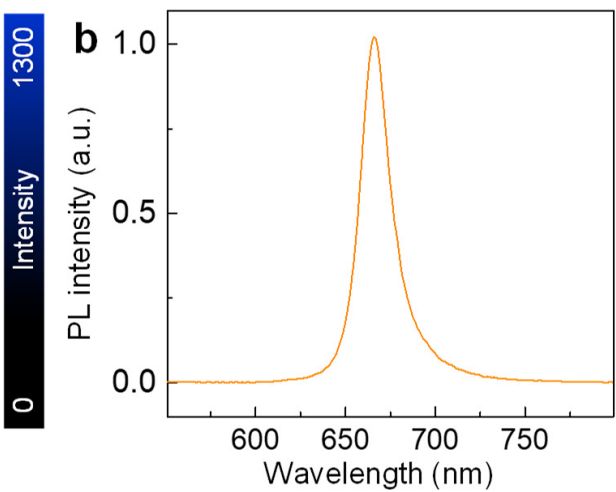

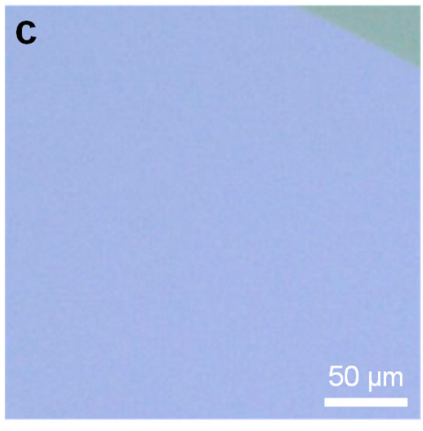

457 Extended Data Fig. 1 | Characterization of the as-grown monolayer $\mathbf{M o S}_{2}$ domain and film.

458 a,b, The PL mapping image (a) and a representative PL spectrum (b) of $\mathrm{MoS}_{2}$ domain grown by 459 S monomer supply. c, The optical image of $\mathrm{MoS}_{2}$ film grown on sapphire by $\mathrm{S}$ monomer supply.

460 The green region on upper right of (c) indicates the bare sapphire substrate. 

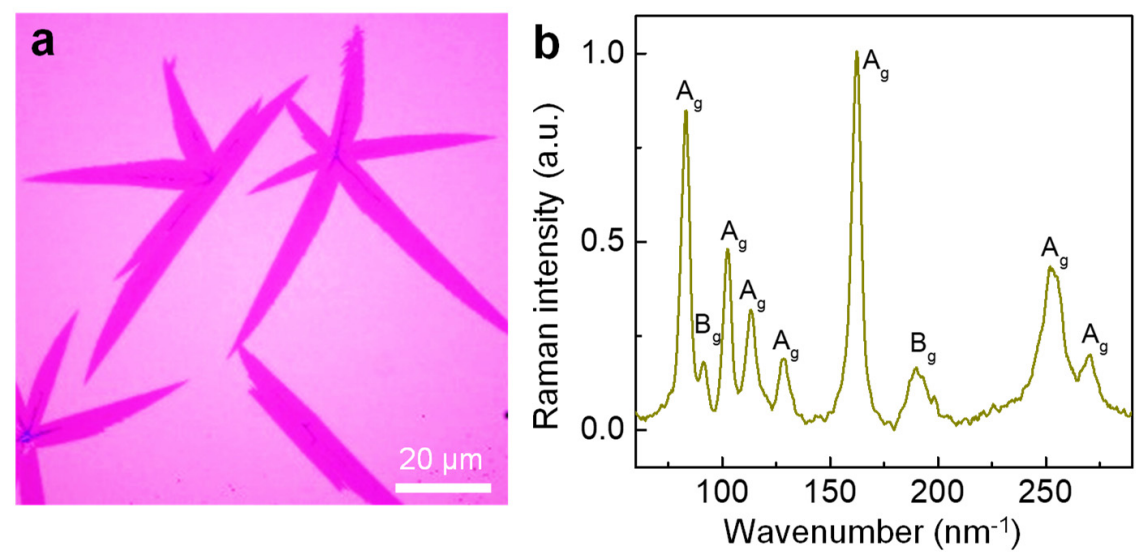

462 Extended Data Fig. 2 | 1T' phase MoTe2 sample prepared by Te monomer supply. a, Optical 463 image of $1 \mathrm{~T}^{\prime}-\mathrm{MoTe}_{2}$. b, Raman spectrum of 1T'-MoTe 2 flake. 

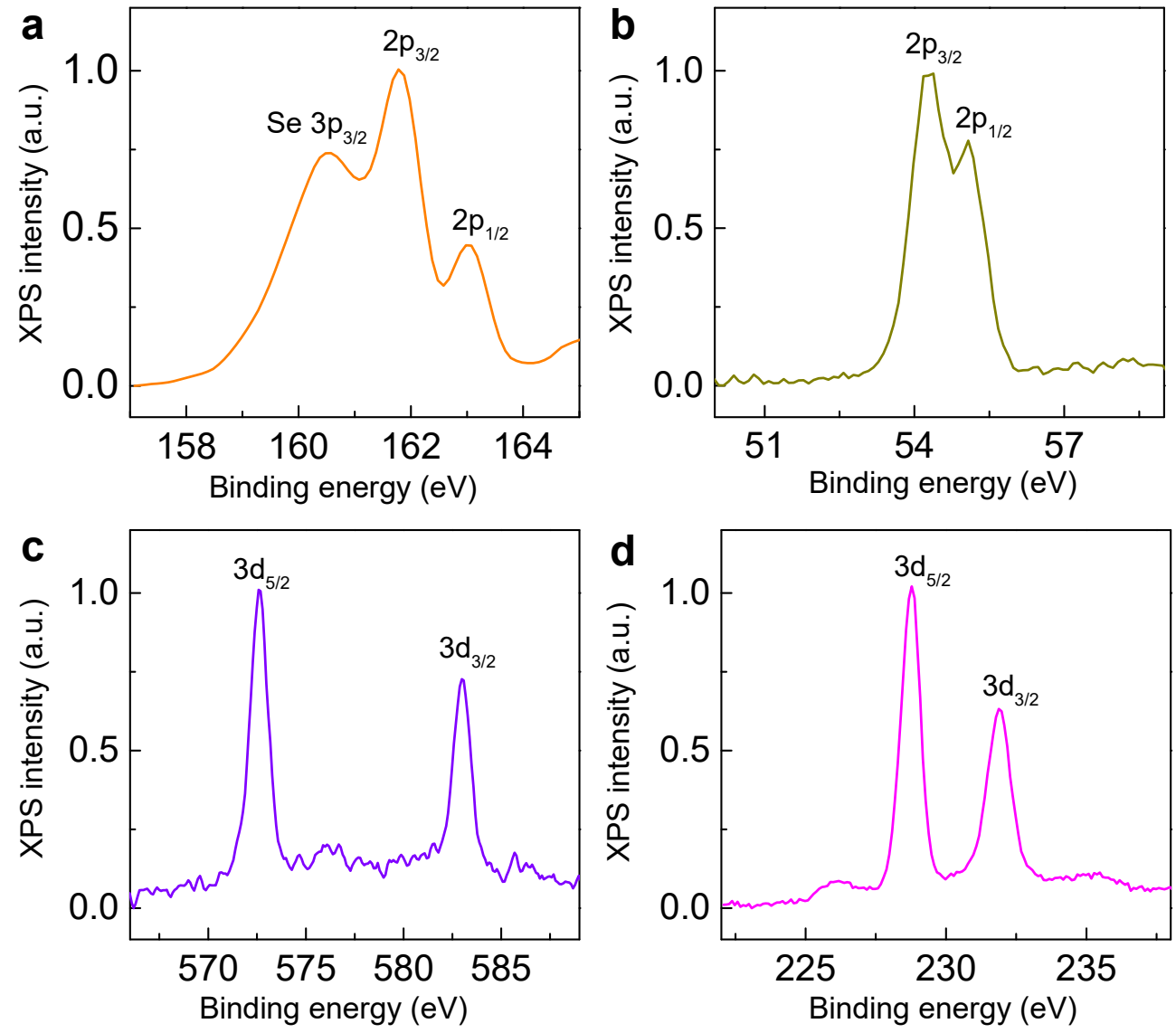

465 Extended Data Fig. 3 | XPS of quaternary 2D $\mathbf{M o S}_{2(1-\mathrm{x}-\mathrm{y})} \mathbf{S e}_{2 \mathrm{x}} \mathbf{T e}_{2 \mathrm{y}}$ alloy. a-d, XPS spectra of 466 as-grown $\mathrm{MoS}_{2(1-x-y)} \mathrm{Se}_{2 x} \mathrm{Te}_{2 y}$ sample, showing binding energy states of Se $3 p$ and $\mathrm{S} 2 \mathrm{p}(\mathrm{a}), \mathrm{Se} 2 \mathrm{p}$ 467 (b), Te 3d (c), and Mo 3d (d), respectively. 

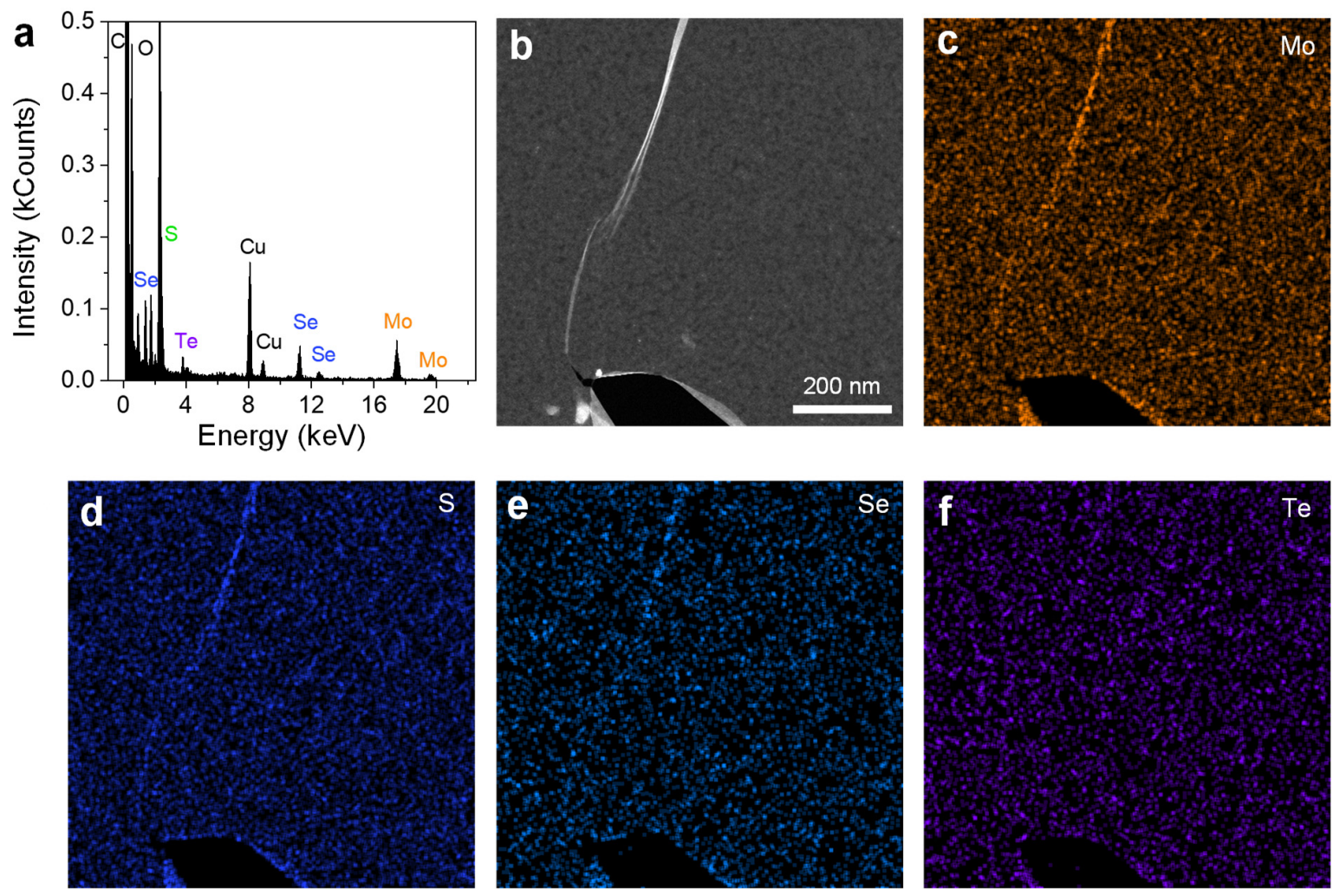

469 Extended Data Fig. 4 | EDS characterization of quaternary monolayer $\mathbf{M o S}_{2(1-\mathrm{x}-\mathrm{y})} \mathrm{Se}_{2 \mathrm{x}} \mathrm{Te}_{2 \mathrm{y}}$

470 alloy. a, EDS spectrum of $\mathrm{MoS}_{2(1-\mathrm{x}-\mathrm{y})} \mathrm{Se}_{2 \mathrm{x}} \mathrm{Te}_{2 \mathrm{y}}$ sample transferred onto TEM $\mathrm{Cu}$ grid. The peaks

471 of S, Se, and Te were clearly observed, and their atomic concentrations were quantitatively

472 extracted to be $84.0 \%, 12.7 \%$, and $3.3 \%$, respectively. $\mathbf{b}$, The scanning transmission electron

473 microscopy (STEM) image of the as-grown monolayer alloy film. c-f, EDS mapping of Mo (c),

$474 \mathrm{~S}(\mathrm{~d}), \mathrm{Se}(\mathrm{e})$, and Te (f) corresponding to the area in (b), showing the uniform element distribution. 

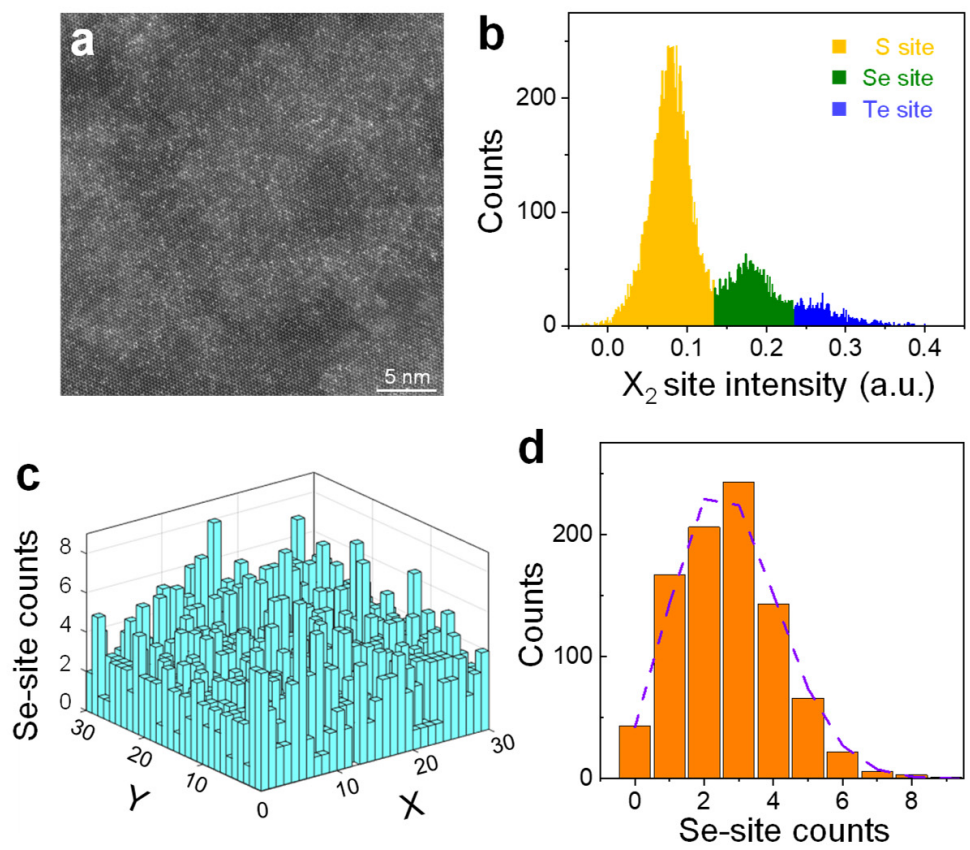

476 Extended Data Fig. 5 | STEM analysis of quaternary $2 D \operatorname{MoS}_{2(1-x-y)} \operatorname{Se}_{2 \mathbf{x}} \mathbf{T e}_{2 y}$ alloy. a, $32 \times 32$

$477 \mathrm{~nm}$ STEM image of $\mathrm{MoS}_{2(1-\mathrm{x}-\mathrm{y})} \mathrm{Se}_{2 x} \mathrm{Te}_{2 y}$ quaternary alloy. The Te-occupied sites in the image

478 exhibit the brightest contrast and uniform distribution. b, Intensity histogram of the chalcogen 479 atom $\left(\mathrm{X}_{2}\right)$ sites in (a). Due to the Z-contrast nature of STEM image, $\mathrm{X}_{2}$ sites occupied by different 480 atoms show distinct intensity ranges. The three peaks are assigned to be "S site", "Se site" and 481 "Te site" regions as marked by yellow, green, and blue, respectively. The statistical result shows $4829122 \mathrm{~S}$ sites, $2417 \mathrm{Se}$ sites, and $771 \mathrm{Te}$ sites from the total $12310 \mathrm{X}_{2}$ sites in (a). c, The Se-site 483 distribution in the divided $30 \times 30$ parts of $(a)$. d, The corresponding statistical histogram of Se484 site counts in each parts of (a). 

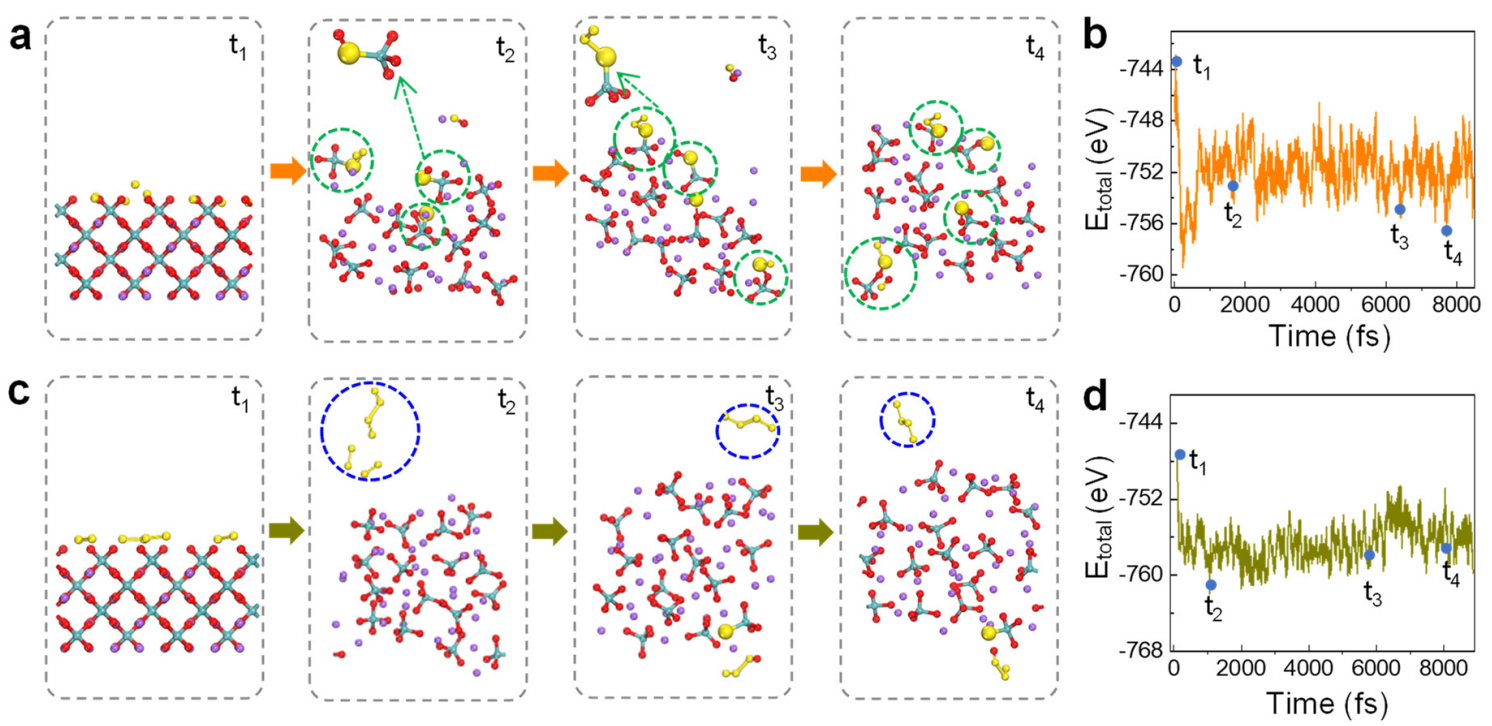

Extended Data Fig. 6 | Snapshots taken during the MD simulation of sulfidation of liquid $\mathrm{Na}_{2} \mathrm{MoO}_{4}$ and the corresponding energy profiles. a,b, Snapshots taken during the MD simulation of sulfidation with eight sulfur monomers and the corresponding energy profiles at $t_{1}$ $(0 \mathrm{ps}), \mathrm{t}_{2}(1.69 \mathrm{ps}), \mathrm{t}_{3}(6.27 \mathrm{ps})$ and $\mathrm{t}_{4}(7.94 \mathrm{ps})$, respectively. It shows that $\mathrm{S}$ monomers can easily substitute the oxygen atoms of $\mathrm{Na}_{2} \mathrm{MoO}_{4}$. c,d, Snapshots taken during the MD simulation of sulfidation with four sulfur dimers and the corresponding energy profiles at $\mathrm{t}_{1}(0 \mathrm{ps}), \mathrm{t}_{2}(1.12 \mathrm{ps})$, $t_{3}(5.97 \mathrm{ps})$ and $t_{4}(8.00 \mathrm{ps})$, respectively. The structures inside green circle present the $\mathrm{MoS}_{x} \mathrm{O}_{4-x}$ cluster, the ones inside blue circle indicate that $\mathrm{S}$ dimers disappears in the form of gas. The yellow, 494 red, violet, and blue spheres represent sulfur, oxygen, sodium and molybdenum atoms, respectively. 

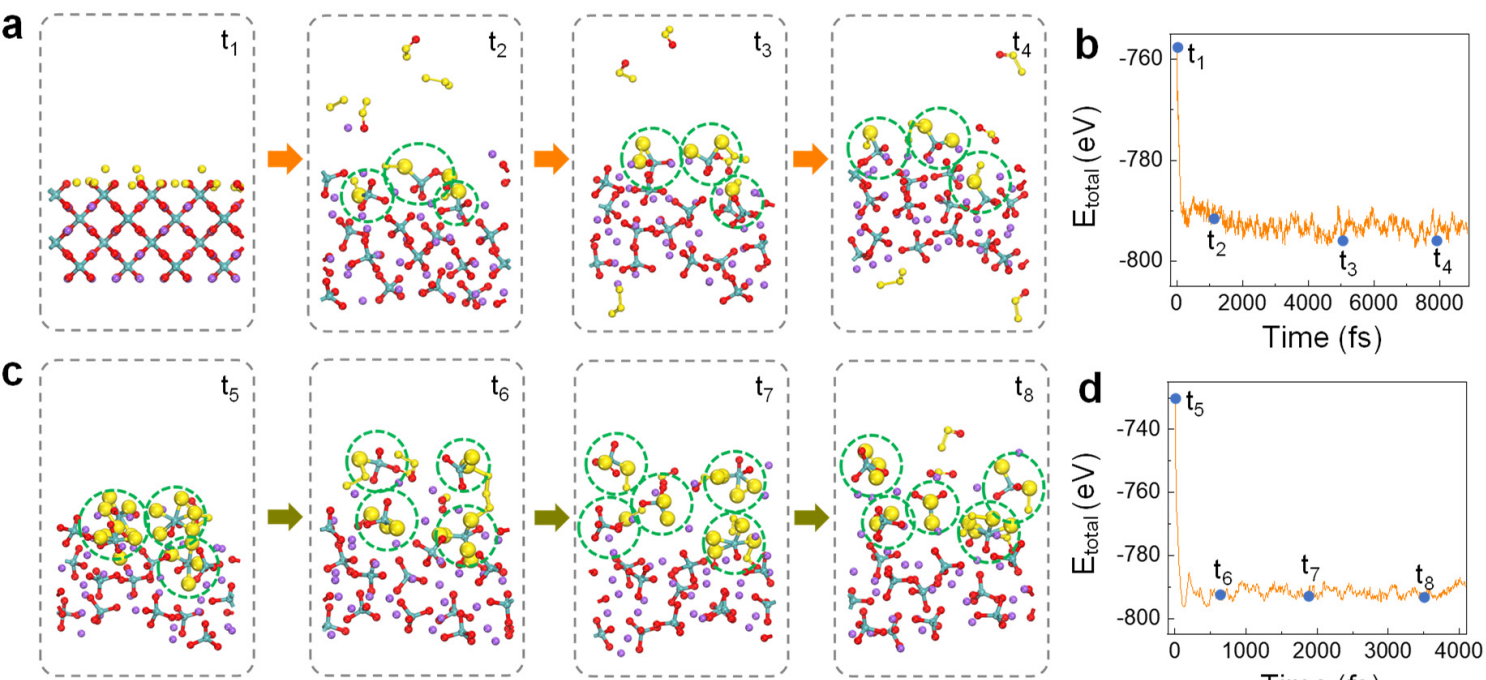

e
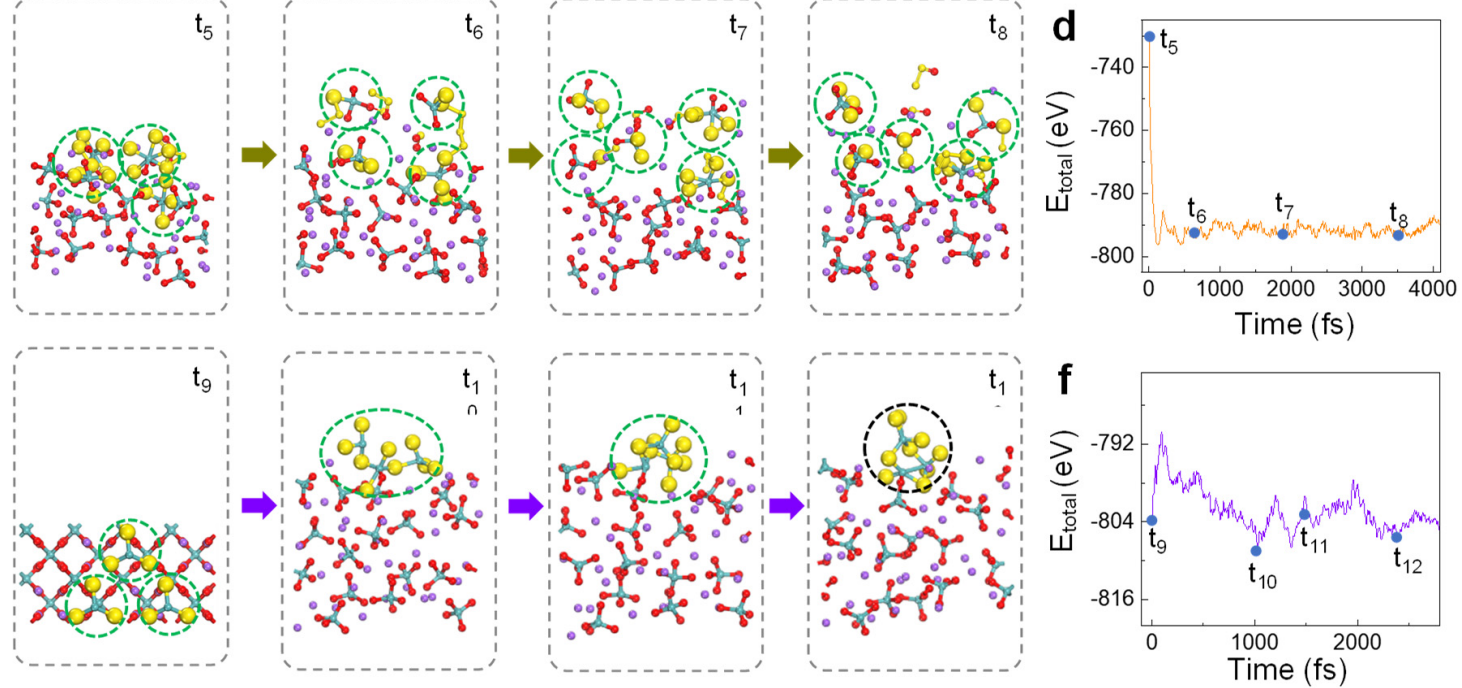

Extended Data Fig. 7 | Snapshots taken during the MD simulation on nucleation process

with S monomers supply and their corresponding energy profiles. a,b, Snapshots taken during the MD simulation when some sulfur monomers exist on the $\mathrm{Na}_{2} \mathrm{MoO}_{4}$ surface in the first stage and the corresponding energy profiles at $\mathrm{t}_{1}(0 \mathrm{ps}), \mathrm{t}_{2}(1.05 \mathrm{ps}), \mathrm{t}_{3}(5.14 \mathrm{ps})$ and $\mathrm{t}_{4}(8.00 \mathrm{ps})$, respectively. c,d, Snapshots taken during the MD simulation when $\mathrm{MoS}_{x} \mathrm{O}_{4-x}$ clusters (green circle) formed on the $\mathrm{Na}_{2} \mathrm{MoO}_{4}$ surface in the second stage and the corresponding energy profiles at $\mathrm{t}_{5}(0 \mathrm{ps}), \mathrm{t}_{6}(0.62 \mathrm{ps}), \mathrm{t}_{7}(1.92 \mathrm{ps})$ and $\mathrm{t}_{8}(3.50 \mathrm{ps})$, respectively. e,f, Snapshots taken during the MD simulation when $\mathrm{Mos}_{x} \mathrm{~S}_{y}$ clusters (green circle) result in the nucleation of $\mathrm{MoS}_{2}$ (black circle) in the finally stage and the corresponding energy profiles at $\mathrm{t}_{9}(0 \mathrm{ps}), \mathrm{t}_{10}(1.0 \mathrm{ps}), \mathrm{t}_{11}(1.50 \mathrm{ps})$ and $\mathrm{t}_{12}$ (2.48 ps), respectively. The yellow, red, violet, and blue spheres represent sulfur, oxygen, sodium, and molybdenum atoms, respectively. 

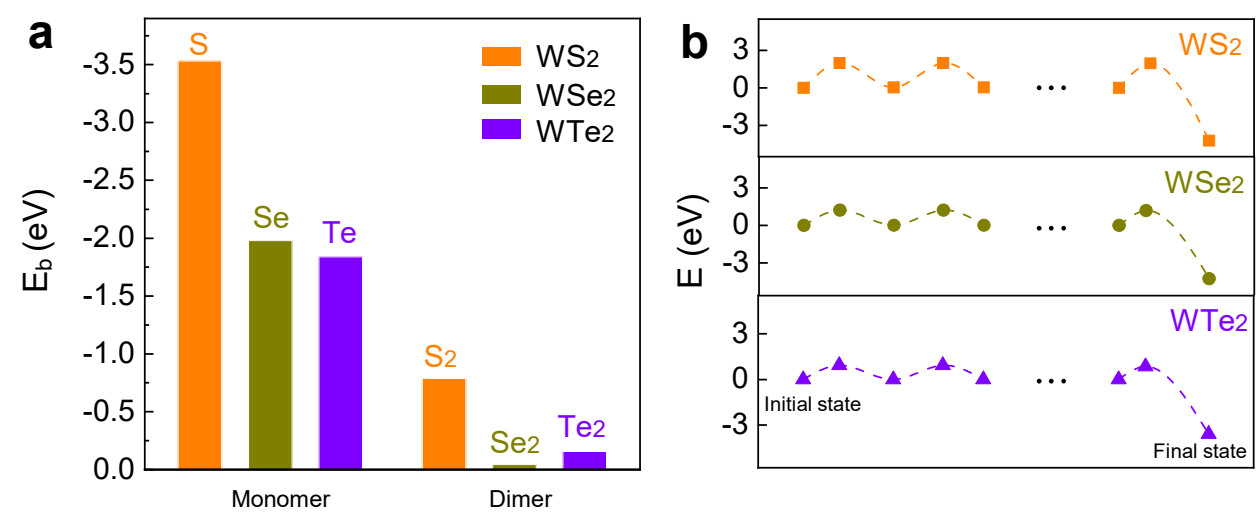

509 Extended Data Fig. 8 | Proposed mechanism of chalcogen monomer modulated $\mathbf{W X}_{2}(\mathbf{X}=$

510 S, Se, Te) growth. a, The binding energies of monomer and dimer on $\mathrm{MoX}_{2}$ surface. b, The 511 energy profiles of vacancy healing on $\mathrm{WX}_{2}$ surface by using chalcogen monomer. 


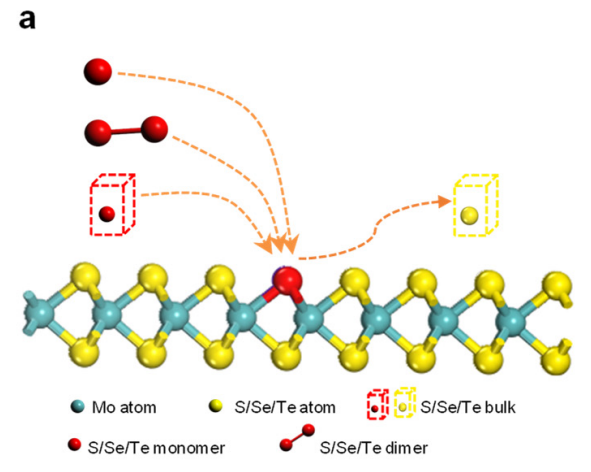

512

513 Extended Data Fig. 9 | Calculated reaction energies of ternary TMD alloys. a, The

514 schematic diagram of substituting a chalcogen atom in a TMD by using chalcogen monomer,

515 dimer and bulk as references. b, The reaction energies to form ternary TMD alloys. It can be

516 clearly seen that the $\mathrm{S}, \mathrm{Se}$, and Te monomers can be easily doped into MoX $\mathrm{X}_{2}$ with reaction 517 energies less than $-2.0 \mathrm{eV}$, while doping of Te dimer or bulk into $\mathrm{MoS}_{2} / \mathrm{MoSe}_{2}, \mathrm{~S}$ bulk into $\mathrm{MoSe}_{2}$,

518 Se bulk into $\mathrm{MoS}_{2}$ are all difficult because of the near-zero or high reaction energies. These results

519 further emphasize the advantage of $\mathrm{S} / \mathrm{Se} / \mathrm{Te}$ monomer supply to grow $\mathrm{MoS}_{2(1-\mathrm{x}-\mathrm{y})} \mathrm{Se}_{2 \mathrm{x}} \mathrm{Te} 2 \mathrm{y}$ alloy. 


\section{Figures}

a
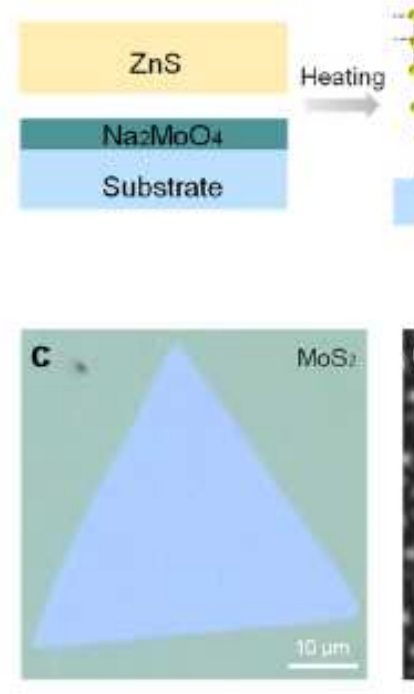
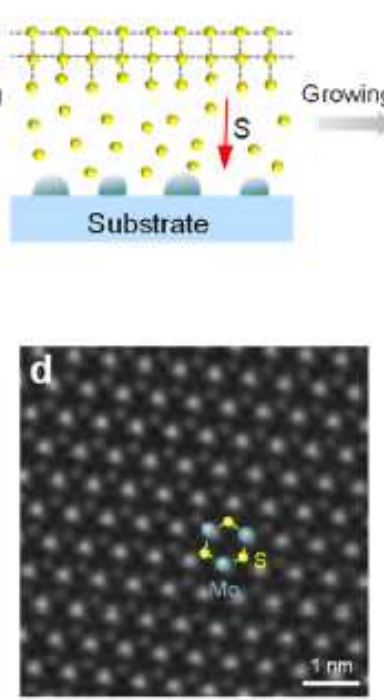

Growing
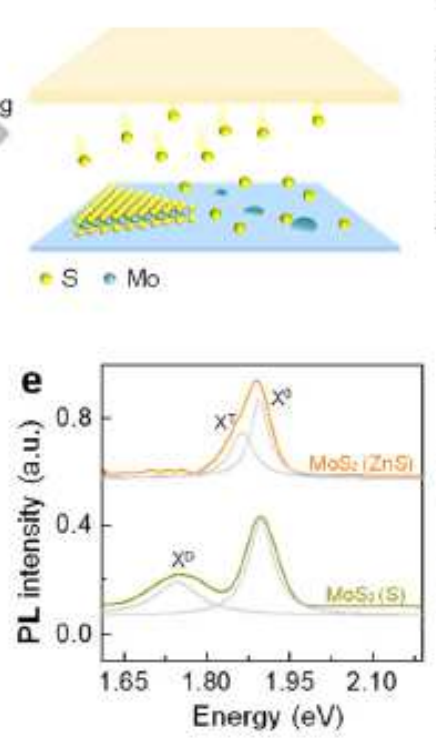
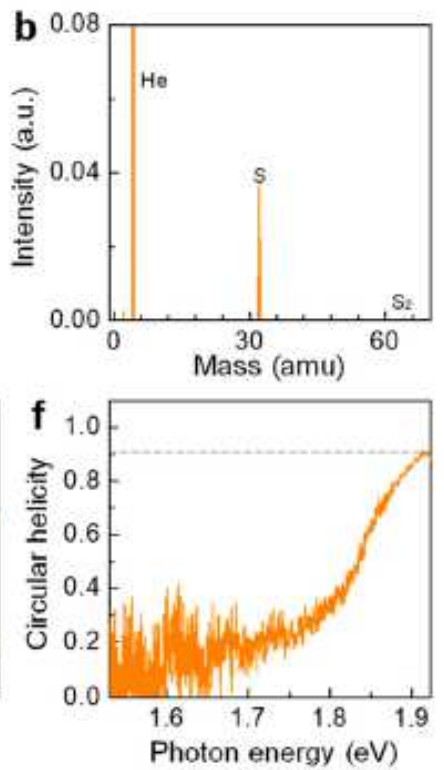

\section{Figure 1}

Growth of high-quality monolayer MoS2 by sulfur monomer supply. a, Schematic of sulfur (S) monomer supply for the growth of MoS2. S monomers are released from the surface of ZnS under high temperature, then confined in the narrow space and react with $\mathrm{Na2MoO} 4$ to from MoS2. b, In-situ mass spectrum of ZnS annealed at 1000 . The intense peak at the mass of 32 clearly proves the dominating release of $\mathrm{S}$ monomers. The measurements were carried out with carrier gas of He and the data was subtracted by background. c, Optical image of as-grown monolayer MoS2 domain on sapphire. d, Atomicresolved HAADF-STEM image of the prepared MoS2, revealing the high crystallinity of MoS2 without detectable $S$ vacancies. e, Low temperature $(10 \mathrm{~K}) \mathrm{PL}$ spectra of MoS2 samples fed by S monomer (orange curve) and $S$ powder (dark yellow curve), respectively. Three typical features, $X 0, X T$, and XD peaks assign to neutral exciton, trion, and defect state emission peaks, respectively. The absence of XD peak confirmed the high quality of MoS2 grown by $S$ monomer supply. $f$, The circular dichroism PL spectrum measured at $10 \mathrm{~K}$. The near-unity polarization of MoS2 on sapphire indicates the high optical quality. 
a
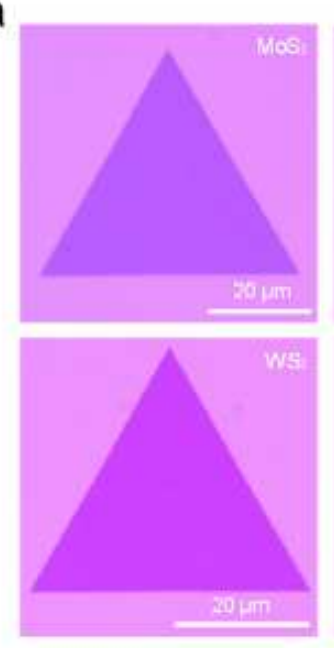

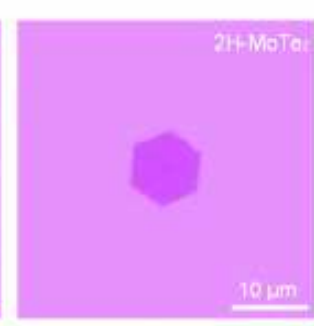

b
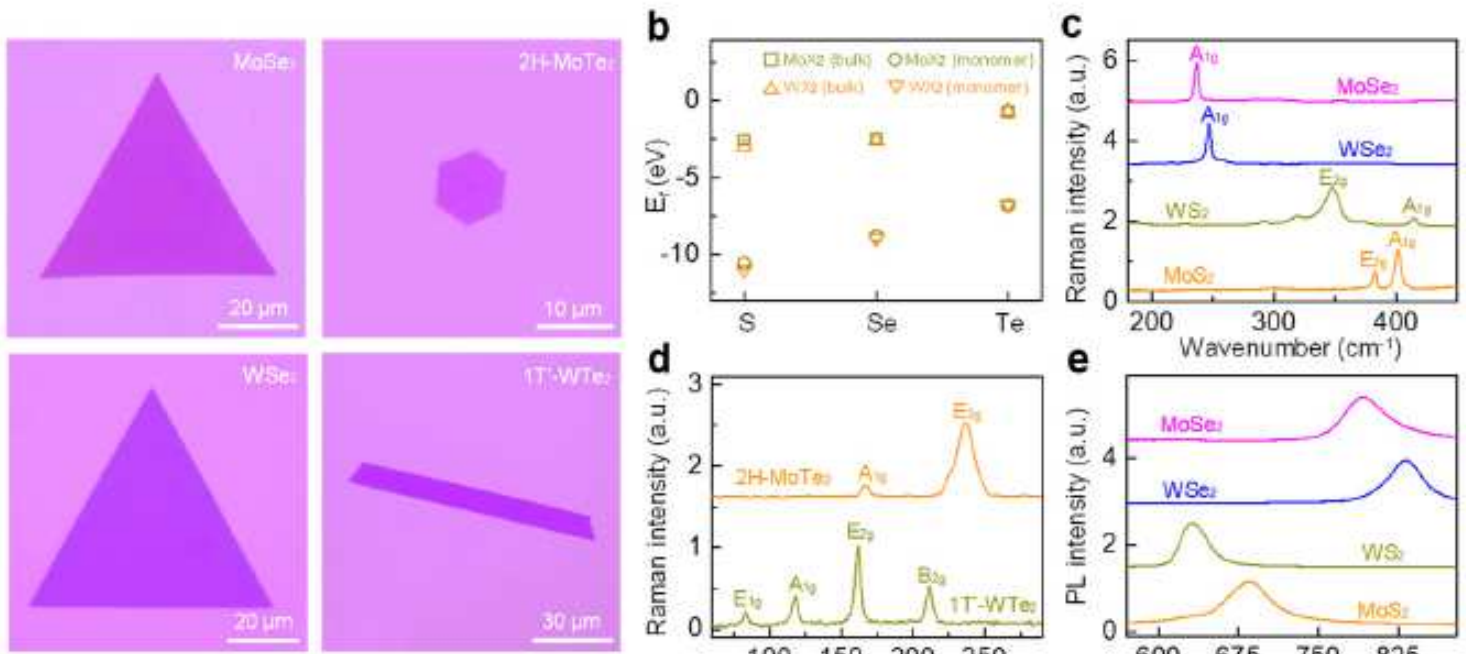

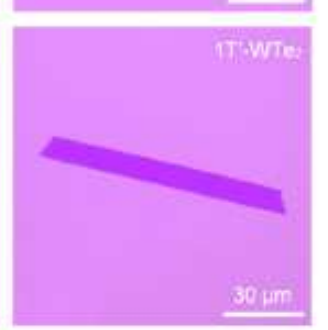

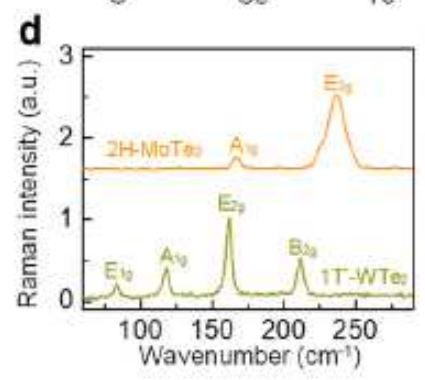

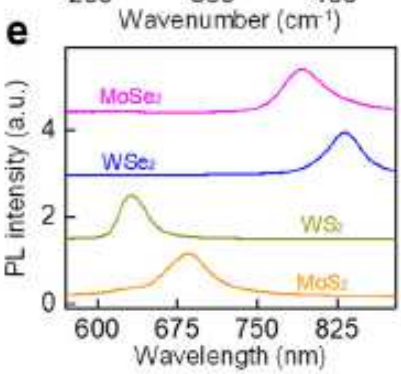

\section{Figure 2}

Universal growth of diverse TMDs by chalcogen monomer supply. a, Optical images of the representative TMDs, including 2H phase MoS2, MoSe2, MoTe2, WS2, WSe2 and 1T' phase WTe2. b, The calculated formation energy (Ef) of the six representative TMDs. When chalcogen bulks are supplied as precursors, the formation of transition metal tellurides in relative to their corresponding sulphide and selenide are less favourable due to their high formation energy (-0.73 and -0.68 eV/unit for MoTe2 and WTe2, respectively). While it becomes highly favourable when Te monomers are applied. c-e, The corresponding Raman (c,d) and PL (e) spectra of TMDs in (a). All spectra were vertically shifted for clarity. 

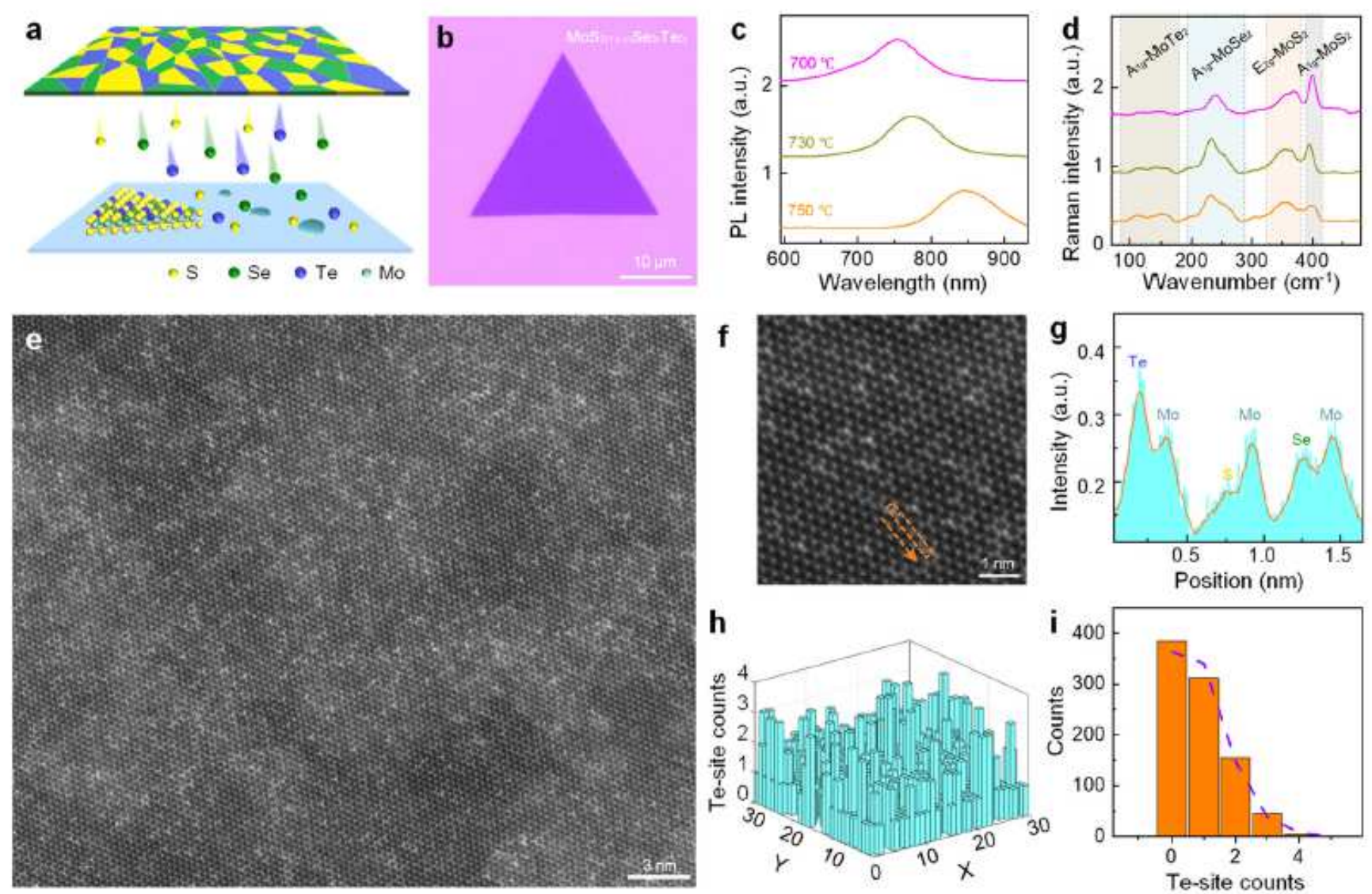

\section{Figure 3}

Growth and characterization of quaternary TMD alloy. a, Schematic diagram of quaternary alloy growth using a compressed plate mixed with chalcogenide powders of ZnS, ZnSe, and ZnTe. b, Optical image of MoS2(1-x-y)Se2xTe2y domain on SiO2/Si substrate. c,d, PL (c) and Raman (d) spectra of the MoS2(1-xy)Se2xTe2y sample grown at different temperatures. As the growth temperature increased, the PL peak position showed a clear red shift. The intensity of MoS2-like E2g $(\sim 380 \mathrm{~cm}-1)$ and A1g $(\sim 400 \mathrm{~cm}-1)$ was reduced while the MoTe2-like A1g ( 150 cm- 1) increased and MoSe2-like A1g ( 240 cm-1) increased first and then reduced. e,f, STEM images of the MoS2(1-x-y)Se2xTe2y, demonstrating the high crystallinity of quaternary alloy. $g$, Intensity profiles along the labeled orange dotted box in ( $f$ ), which highlights the occupancies of Mo, S, Se, and Te sites. h, The Te-site distribution in a $32 \times 32 \mathrm{~nm}$ STEM image of the quaternary alloy. The image was divided into $30 \times 30$ parts. i, The corresponding statistical histogram of Te-site counts in each parts of the image. It shows a well binomial distribution feature (purple dotted line), revealing the random distribution of Te atoms. 

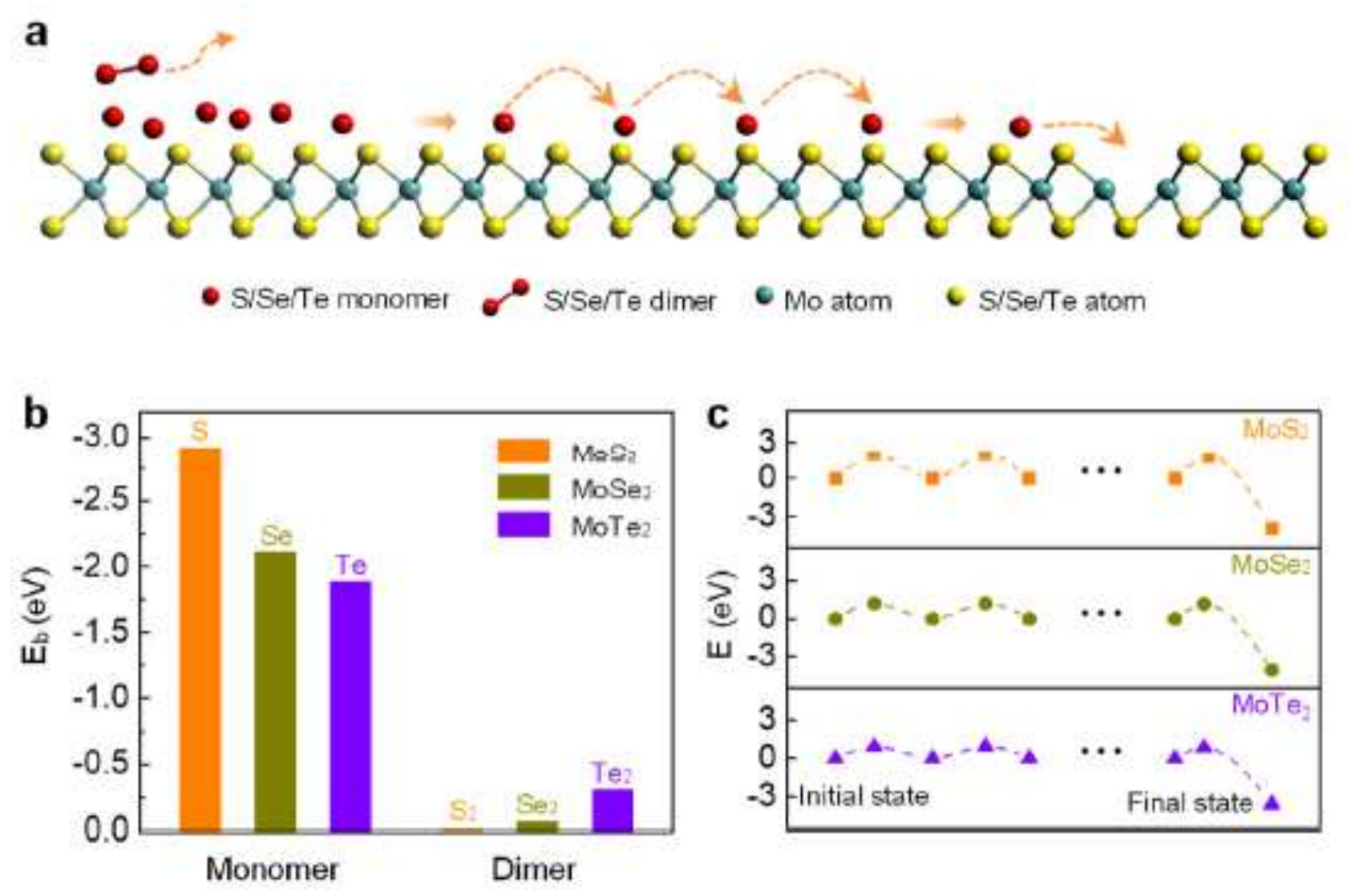

\section{Figure 4}

Growth Mechanism with chalcogen monomer supply in MoX2 (X = S, Se, Te). a, Schematic diagram of adsorption, diffusion, and vacancy healing of chalcogen monomer on MoX2 surface. $b$, The binding energies of monomers and dimers on MoX2 surface. The much higher energy of monomers facilitates their better adsorption on the TMD surface than dimers. c, The energy profiles of vacancy healing for MoX2 surface by using chalcogen monomers. The relatively small energy barriers of chalcogen monomer diffusion and the highly exothermic reaction at the vacancy both accelerate the self-healing of MoX2. 